\title{
Imidazolium-based ionic liquids with large weakly coordinating anions
}

\author{
William Levason, David Pugh* and Gillian Reid
}

Chemistry, University of Southampton, Highfield, Southampton, SO17 1BJ, UK

\begin{abstract}

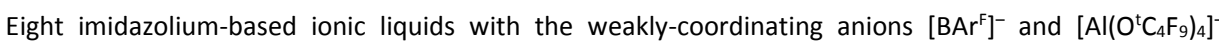
$\left([\mathrm{BArF}]^{-}=\right.$tetrakis\{3,5-bis(trifluoromethyl)phenyl\}borate) have been synthesized. Characterization by ${ }^{1} \mathrm{H}$ NMR spectroscopy shows that the salts are fully dissociated in solution. Six examples have been further characterized by X-ray crystallography, revealing that weak hydrogen bonds do occur in the solid phase between the imidazolium cations and weakly coordinating anions. Comparison with the bulky [IDiPPH] imidazolium cation (IDiPPH = 1,3-bis\{2,6-bis(diisopropyl)phenyl\}imidazolium) shows that large steric bulk close to the imidazolium protons can preclude hydrogen bonds from forming. Differential scanning calorimetry of the salts reveal that all are thermally stable up to $200{ }^{\circ} \mathrm{C}$ which renders them as potentially suitable background electrolytes for electrochemical processes which take place at elevated temperatures.
\end{abstract}

\section{Introduction}

It has long been known that "non-coordinating" anions such as $\left[\mathrm{BF}_{4}\right]^{-}$and $\left[\mathrm{PF}_{6}\right]^{-}$are capable of interacting with electrophilic metal centres, thus they are more accurately referred to as weakly coordinating anions (WCAs). ${ }^{1}$ More recent efforts towards developing truly non-coordinating anions led to the synthesis of fluorinated tetrakis(aryl)borates, such as $\left[\mathrm{B}\left\{3,5-\left(\mathrm{CF}_{3}\right)_{2} \mathrm{C}_{6} \mathrm{H}_{3}\right\}_{4}\right]^{-}$(hereafter $[\mathrm{BAr}]^{-}$), and fluorinated tetrakis(alkoxy)aluminates, such as $\left[\mathrm{Al}\left(\mathrm{O}^{t} \mathrm{C}_{4} \mathrm{~F}_{9}\right)_{4}\right]^{-2}$. These large, highly fluorinated anions are ideal for stabilising very reactive metal fragments such as sigma complexes of alkanes with transition metals ${ }^{3}$ and p-block elements with low oxidation states or coordination numbers. ${ }^{4}$ We have recently used the $\left[\mathrm{BAr}^{\mathrm{F}}\right]^{-}$and $\left[\mathrm{Al}\left(\mathrm{O}^{\mathrm{t}} \mathrm{C}_{4} \mathrm{~F}_{9}\right)_{4}\right]^{-}$WCAs to isolate unusual coordination compounds of the s-block cations, including 'sandwich' complexes of $\mathrm{Na}^{+}$and $\mathrm{K}^{+}$with aza-macrocycles, homoleptic phosphane complexes of $\mathrm{Li}^{+}$and $\mathrm{Na}^{+}$, and homoleptic octathioether coordination to $\mathrm{Na}^{+}{ }^{5}$ It is notable that these large WCAs are still not truly non-coordinating because the $\left[\mathrm{Al}\left(\mathrm{O}^{t} \mathrm{C}_{4} \mathrm{~F}_{9}\right)\right]^{-}$anion has been observed to interact with metals such as $\mathrm{Ag}^{2 \mathrm{~b}}$ and we observed significant interactions between the $\left[\mathrm{BAr}{ }^{\mathrm{F}}\right]^{-}$anion and the Group 1 cations. ${ }^{6}$

Supercritical fluid electrodeposition (SCFED) is under development as a method for depositing thin films and nanostructures of technologically relevant materials. ${ }^{7}$ SCFED exploits the unique properties of supercritical fluids, namely: high mass transport in a fluid which has no surface tension, to penetrate extreme nanopores ( $<3 \mathrm{~nm}$ diameter) and electrodeposit materials such as $\mathrm{Cu}^{8}$ Amongst the precursors found to be suitable for this work in supercritical $\mathrm{CH}_{2} \mathrm{~F}_{2}$, halometallate salts of the desired material feature strongly, e.g. $\left[\mathrm{N}^{\mathrm{n}} \mathrm{Bu}_{4}\right]\left[\mathrm{GeCl}_{3}\right]$ to deposit $\mathrm{Ge} .{ }^{9}$ Owing to the low polarity of the supercritical fluids, a

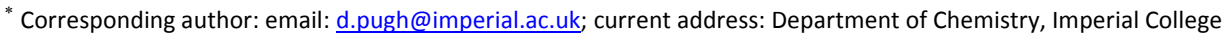
London, South Kensington, London, SW7 2AZ, UK
} 
charge-carrying background electrolyte is required to provide conductivity in the system. Typically, the electrolyte is a salt with a WCA (or halide anion) which is cation-matched to the precursor (i.e. for the $\left[\mathrm{N}^{\mathrm{n}} \mathrm{Bu}_{4}\right]\left[\mathrm{GeCl}_{3}\right]$ precursor, the background electrolyte could be $\left[\mathrm{N}^{\mathrm{n}} \mathrm{Bu}_{4}\right]\left[\mathrm{BF}\right.$ ] or $\left.\left[\mathrm{N}^{\mathrm{n}} \mathrm{Bu} 4\right] \mathrm{Cl}\right)$. A study by Nishi et al. showed that the WCA salts possessed a wide electrochemical window under two-phase water-ionic liquid conditions. This renders them potentially useful charge-carrying reagents for SCFED purposes. ${ }^{10}$ Subsequent investigations into the solubility and conductivity of the $[\mathrm{BAr}]^{-}$and $\left[\mathrm{Al}\left(\mathrm{O}^{\mathrm{t}} \mathrm{C}_{4} \mathrm{~F}_{9}\right)_{4}\right]^{-}$salts were carried out in several supercritical fluids, revealing that they were significantly more conducting than electrolytes with smaller WCAs such as $\left[\mathrm{BF}_{4}\right]^{-}$and $\left[\mathrm{NTf}_{2}\right]^{-}$at similar molar concentrations. ${ }^{11}$

Imidazolium-based cations such as [EMIM] $]^{+}$(1-ethyl-3-methylimidazolium) typically exhibit solid-state hydrogen bonds towards halometallate anions, and these are often retained in solution. ${ }^{12}$ These interactions also appear to influence the resulting electrodeposited film. In a recent study we found that Ge precursors containing [EMIM] ${ }^{+}$cations were more likely to result in the deposition of protocrystalline Ge, compared to precursors containing $\left[\mathrm{N}^{\mathrm{n}} \mathrm{Bu}_{4}\right]^{+}$cations. ${ }^{13}$ Therefore, imidazolium-based halometallate salts are an interesting class of precursor for SCFED and suitable cation-matched background electrolytes are needed.

Imidazolium-based ionic liquids with WCAs have been proven to be versatile reagents, for example the hydrovinylation of styrene $\mathrm{e}^{14 \mathrm{a}}$ and hydrogenation of imines ${ }^{14 \mathrm{~b}}$ were carried out in high yield when using [EMIM][BAr ${ }^{\mathrm{F}}$ ] as a solvent. Imidazolium-based ionic liquids with the [BAr $\left.{ }^{\mathrm{F}}\right]^{-}$anion have also been shown to act as a catalyst for the Diels-Alder reaction and as stoichiometric additives in Ni-catalyzed ethylene oligomerization. ${ }^{14 c, d}$ Although several examples have previously been synthesized, data on the cationanion interactions is very limited, mainly because crystallographic data on these compounds is sparse. Only two solid state structures of imidazolium salts with [BArF] ${ }^{-}$are known, namely [BMIM][BAr ${ }^{\mathrm{F}}$ (BMIM $=$ 1-butyl-3-methylimidazolium) and $[\mathrm{IMesH}]\left[\mathrm{BAr}{ }^{\mathrm{F}}\right] \quad$ (IMesH $\quad=\quad 1,3$-bis $(2,4,6-$ trimethylphenyl)imidazolium), ${ }^{15 a, b}$ and there are no structurally characterized examples with the $\left[\mathrm{Al}\left(\mathrm{O}^{t} \mathrm{C}_{4} \mathrm{~F}_{9}\right)_{4}\right]^{-}$anion (a few structures are known with the related $\left[\mathrm{Al}\left(\mathrm{OCH}\left\{\mathrm{CF}_{3}\right\}_{2}\right)_{4}\right]^{-}$anion). ${ }^{15 c-e}$

In this paper we report the synthesis of eight imidazolium salts comprising the [EMIM] $]^{+},[\mathrm{HMIM}]^{+}$, [EDMIM $^{+}$and $[\mathrm{EMBIM}]^{+}$cations with $[\mathrm{BAr}]^{-}$and $\left[\mathrm{Al}\left(\mathrm{O}^{\mathrm{t}} \mathrm{C}_{4} \mathrm{~F}_{9}\right)_{4}\right]^{-}$anions (Figure 1$)$. All have been characterized by NMR spectroscopy $\left({ }^{1} \mathrm{H},{ }^{13} \mathrm{C}\left\{{ }^{1} \mathrm{H}\right\}\right.$ and $\left.{ }^{19} \mathrm{~F}\left\{{ }^{1} \mathrm{H}\right\}\right)$, differential scanning calorimetry (DSC) and microanalysis, and six examples have also been structurally characterized. For comparison purposes we also report the synthesis and structural characterization of [IDiPPH][BArF] (IDiPPH $=1,3-b i s(2,6-$ diisopropylphenyl)imidazolium), which is a very bulky imidazolium salt containing acidic protons, as well as [BMPYRR][BArF ${ }^{\mathrm{F}}$ (BMPYRR = 1-butyl-1-methylpyrrolidinium) which does not contain any acidic protons.

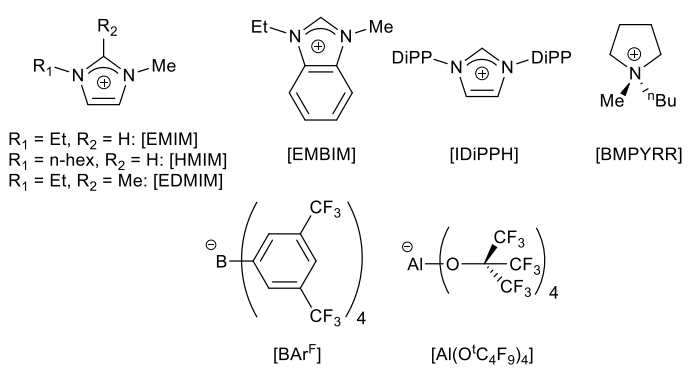

Figure 1: the cations (top row) and anions (bottom row) used in this paper. DiPP = 1,3-diisopropylphenyl. 


\section{Experimental}

All preparations were carried out in air at room temperature using a slight modification of the procedure reported by Kühn and co-workers. ${ }^{16}$ The salts of the $\left[\mathrm{Al}\left(\mathrm{O}^{\mathrm{t}} \mathrm{C}_{4} \mathrm{~F}_{9}\right)_{4}\right]^{-}$anion were mildly hygroscopic and were stored in a glove box. [EMIM]Br, [EDMIM]Cl, [HMIM]Cl and [BMPYRR]Br were purchased from Sigma and used as received. $\mathrm{Li}\left[\mathrm{Al}\left(\mathrm{O}^{\mathrm{t}} \mathrm{C}_{4} \mathrm{~F}_{9}\right)_{4}\right], \mathrm{Na}$ [BAr $\left.{ }^{\mathrm{F}}\right],[\mathrm{EMBIM}] \mathrm{I}$ and [IDiPPH]Cl were synthesized by literature methods. ${ }^{1 b, 17}{ }^{1} \mathrm{H},{ }^{13} \mathrm{C}\left\{{ }^{1} \mathrm{H}\right\}$ and ${ }^{19} \mathrm{~F}\left\{{ }^{1} \mathrm{H}\right\} \mathrm{NMR}$ spectra were recorded in $\mathrm{CDCl}_{3},\left(\mathrm{CD}_{3}\right)_{2} \mathrm{CO}$ or $\mathrm{CD}_{2} \mathrm{Cl}_{2}$ solution at $298 \mathrm{~K}$ using a Bruker $\mathrm{AV}-300$ or $\mathrm{AV}-400$ spectrometer and are referenced to the residual protio-solvent resonance $\left({ }^{1} \mathrm{H},{ }^{13} \mathrm{C}\left\{{ }^{1} \mathrm{H}\right\}\right)$ or $\mathrm{CFCl}_{3}\left({ }^{19} \mathrm{~F}\left\{{ }^{1} \mathrm{H}\right\}\right)$. Microanalyses were undertaken by Medac Ltd. and London Metropolitan University.

DSC was carried out on a TA Instruments Discovery DSC operated by Trios software. Data analysis was also carried out using Trios. Crystalline samples, as obtained below, were heated to $200{ }^{\circ} \mathrm{C}$ at a rate of $10{ }^{\circ} \mathrm{C} / \mathrm{min}$, then cooled to $-50{ }^{\circ} \mathrm{C}$ at $10{ }^{\circ} \mathrm{C} / \mathrm{min}$ and heated back to $200{ }^{\circ} \mathrm{C}$ at $10{ }^{\circ} \mathrm{C} / \mathrm{min}$ (apart from [EMBIM] $\left[\mathrm{Al}\left(\mathrm{O}^{\mathrm{t}} \mathrm{C}_{4} \mathrm{~F}_{9}\right)_{4}\right]$ and [EDMIM] $\left[\mathrm{Al}\left(\mathrm{O}^{\mathrm{t}} \mathrm{C}_{4} \mathrm{~F}_{9}\right)_{4}\right]$ which were heated to $\left.250^{\circ} \mathrm{C}\right)$.

X-Ray crystallography: Crystals were obtained as described below. Details of the crystallographic data collection and refinement are in Table 1. Diffractometer: Rigaku AFC12 goniometer equipped with an enhanced sensitivity (HG) Saturn724+ detector mounted at the window of an FR-E+ SuperBright molybdenum rotating anode generator $\left(\lambda_{1}=0.71073 \AA\right.$ A ) with VHF Varimax optics (70 or $110 \mu \mathrm{m}$ focus). Cell determination and data collection: CrystalClear-SM Expert 3.1 b27, data reduction, cell refinement, and absorption correction: CrystalClear-SM Expert 2.1. ${ }^{18}$ Structure solution and refinement were carried out using WinGX and software packages within. ${ }^{19}$ Disorder in the $\mathrm{CF}_{3}$ groups of the $\left[\mathrm{BAr} \mathrm{F}^{-}\right.$anions was present in all of the structures, which is often observed in compounds containing $\left[\mathrm{BAr}^{\mathrm{F}}\right]^{-},{ }^{20}$ and this was satisfactorily modelled using DFIX, DANG, ISOR, DELU, and SIMU restraints. The [EDMIM] ${ }^{+}$cations were positionally disordered over an inversion centre which necessitated the use of PART -1 instructions. $\mathrm{H}-$ atoms were placed in geometrically-assigned positions with $\mathrm{C}-\mathrm{H}$ distances of $0.95 \AA(\mathrm{CH}), 0.98 \AA\left(\mathrm{CH}_{3}\right)$ or $0.99 \AA\left(\mathrm{CH}_{2}\right)$ and refined using a riding model with $\mathrm{U}_{\text {iso }}(\mathrm{H})=1.2 \mathrm{U}_{\text {eq }}(\mathrm{C})\left(\mathrm{CH}, \mathrm{CH}_{2}\right)$ or $1.5 \mathrm{U}_{e q}(\mathrm{C})\left(\mathrm{CH}_{3}\right)$. enClFer was used to prepare material for publication. ${ }^{21}$ CCDC reference numbers 1514180 ([EMIM][BAr $\left.{ }^{\mathrm{F}}\right]$ ), 1514181 ([EDMIM][BAr $\left.\left.{ }^{F}\right]\right), \quad 1514182$ ([HMIM][BAr $\left.\left.{ }^{\mathrm{F}}\right]\right), \quad 1514183$ ([BMPYRR][BAr $\left.\left.{ }^{\mathrm{F}}\right]\right), \quad 1514184$ ([EMBIM][BAr $\left.\left.{ }^{\mathrm{F}}\right]\right), \quad 1514185 \quad$ ([IDiPPH][BAr $\left.\left.{ }^{\mathrm{F}}\right]\right), \quad 1514186 \quad\left([\mathrm{EMIM}]\left[\mathrm{Al}\left(\mathrm{O}^{\mathrm{t}} \mathrm{C}_{4} \mathrm{~F}_{9}\right)_{4}\right]\right)$ and 1514187 ([EMBIM] $\left.\left[\mathrm{Al}\left(\mathrm{O}^{\mathrm{t}} \mathrm{C}_{4} \mathrm{~F}_{9}\right)_{4}\right]\right)$ contain crystallographic data in CIF format.

[EMIM][BAr']: [EMIM]Br (108 mg, $0.56 \mathrm{mmol})$ and $\mathrm{Na}\left[\mathrm{BAr} \mathrm{F}^{\mathrm{F}}\right.$ ] $(500 \mathrm{mg}, 0.56 \mathrm{mmol})$ were suspended in acetone $(20 \mathrm{~mL})$ and stirred for four hours. After filtration through Celite ${ }^{\circledR}$, the volatiles were removed, leaving a sticky off-white solid. This was dissolved in $\mathrm{CH}_{2} \mathrm{Cl}_{2}$ and filtered through a short plug of silica, then crystallization occurred from vapour diffusion of $\mathrm{Et}_{2} \mathrm{O}$ into a concentrated $\mathrm{CH}_{2} \mathrm{Cl}_{2}$ solution. Yield: 317 $\mathrm{mg}$, 58\%. Anal. calc. for $\mathrm{C}_{38} \mathrm{H}_{23} \mathrm{~N}_{2} \mathrm{BF}_{24}$ (974.15): C 46.84; H 2.38; N 2.88. Found C 46.90; $\mathrm{H} 2.95 ; \mathrm{N} 2.99 .{ }^{1} \mathrm{H}$ $\operatorname{NMR}\left(400.1 \mathrm{MHz}, \mathrm{CDCl}_{3}\right): 8.74(\mathrm{~s},[1 \mathrm{H}], \mathrm{H} 2), 7.69$ (s, [8H], BArF H2/6), $7.53\left(\mathrm{~s},[4 \mathrm{H}], \mathrm{BAr}^{\mathrm{F}} \mathrm{H} 4\right), 7.08,7.02$ (each s, $[1 \mathrm{H}], \mathrm{H} 4 / 5), 4.10\left(\mathrm{q}, J=7.1 \mathrm{~Hz},[2 \mathrm{H}], \mathrm{CH}_{2}\right), 3.79\left(\mathrm{~s},[3 \mathrm{H}], \mathrm{NCH}_{3}\right), 1.46\left(\mathrm{t}, J=7.6 \mathrm{~Hz},[3 \mathrm{H}], \mathrm{CH}_{3}\right) \mathrm{ppm}$. (400.1 MHz, CD $\mathrm{Cl}_{2}$ ): $8.10(\mathrm{~s},[1 \mathrm{H}], \mathrm{H} 2), 7.72\left(\mathrm{~s},[8 \mathrm{H}], \mathrm{BAr}^{\mathrm{F}} \mathrm{H} 2 / 6\right), 7.56$ (s, [4H], BAr $\left.{ }^{\mathrm{F}} \mathrm{H} 4\right), 7.29,7.25$ (each s, $[1 \mathrm{H}], \mathrm{H} 4 / 5), 4.19$ (q, J = 7.3 Hz, [2H], NCH 2$), 3.89\left(\mathrm{~s},[3 \mathrm{H}], \mathrm{NCH}_{3}\right), 1.55\left(\mathrm{t}, J=7.3 \mathrm{~Hz}, \mathrm{CH}_{3}\right) .{ }^{13} \mathrm{C}\left\{{ }^{1} \mathrm{H}\right\} \mathrm{NMR}$ (100.6 MHz, CDCl $)_{3}$ : 161.63 (C, q, $\mathrm{J}_{\mathrm{C}-\mathrm{B}}=50.3 \mathrm{~Hz}, \mathrm{BAr} \mathrm{F}^{\mathrm{F}} \mathrm{C}$ ), 134.68 (CH, BArF C2/6), 128.81 (C, q, $\mathrm{J}_{\mathrm{C}-\mathrm{F}}=2.9 \mathrm{~Hz}$, $\left.\mathrm{BAr}^{\mathrm{F}} \mathrm{C} 3 / 5\right), 124.44\left(\mathrm{C}, \mathrm{q}, \mathrm{J}_{\mathrm{C}-\mathrm{F}}=272 \mathrm{~Hz}, \mathrm{CF}_{3}\right.$ ), 123.57, 121.98 (each CH, C4/5), 117.50 (CH, BArF C4), 45.58 $\left(\mathrm{CH}_{2}\right), 36.30\left(\mathrm{NCH}_{3}\right), 14.77\left(\mathrm{CH}_{3}\right) \mathrm{ppm} .{ }^{19} \mathrm{~F}\left\{{ }^{1} \mathrm{H}\right\} \mathrm{NMR}\left(282.4 \mathrm{MHz}, \mathrm{CDCl}_{3}\right):-62.70 \mathrm{ppm}$.

[EDMIM][BAr']: [EDMIM]Cl $(90 \mathrm{mg}, 0.56 \mathrm{mmol})$ and $\mathrm{Na}$ [BAr ${ }^{\mathrm{F}}$ ] $(500 \mathrm{mg}, 0.56 \mathrm{mmol})$ were suspended in acetone $(20 \mathrm{~mL})$ and stirred for four hours. After filtration through Celite ${ }^{\varpi}$, the volatiles were removed, 
leaving a sticky off-white solid. This was redissolved in $\mathrm{CH}_{2} \mathrm{Cl}_{2}$, filtered through a short plug of silica and the $\mathrm{CH}_{2} \mathrm{Cl}_{2}$ was removed in vacuo, affording $317 \mathrm{mg}$ of a white solid in $58 \%$ yield. Crystallization occurred from slow evaporation of a $\mathrm{CH}_{2} \mathrm{Cl}_{2}$ solution. Anal. calc. for $\mathrm{C}_{39} \mathrm{H}_{25} \mathrm{~N}_{2} \mathrm{BF}_{24}$ (988.16): $\mathrm{C} 47.36 ; \mathrm{H} 2.55 ; \mathrm{N} 2.83$. Found C 47.08; H 2.14; N 3.35. ${ }^{1} \mathrm{H}$ NMR (300.0 MHz, CDCl $)$ : 7.69 (s, [8H], BAr $\left.{ }^{\mathrm{F}} \mathrm{H} 2 / 6\right), 7.53$ (s, [4H], BAr $\mathrm{H} 4$ ), 6.91, 6.84 (each d, $J=2.2 \mathrm{~Hz},[1 \mathrm{H}]$, EDMIM H4/5), 3.90 (q, $J=7.7 \mathrm{~Hz},[2 \mathrm{H}], \mathrm{CH}_{2}$ ), $3.54\left(\mathrm{~s},[3 \mathrm{H}], \mathrm{NCH}_{3}\right.$ ), $2.39\left(\mathrm{~s},[3 \mathrm{H}], \mathrm{CCH}_{3}\right), 1.34\left(\mathrm{t}, J=7.7 \mathrm{~Hz},[3 \mathrm{H}], \mathrm{CH}_{3}\right)$ ppm. (400.1 MHz, $\mathrm{CD}_{2} \mathrm{Cl}_{2}$ ): $7.72\left(\mathrm{~s},[8 \mathrm{H}], \mathrm{BAr}^{\mathrm{F}} \mathrm{H} 2 / 6\right), 7.56$ (s, [4H], BAr $\left.{ }^{\mathrm{F}} \mathrm{H} 4\right), 7.16,7.12$ (each d, $J=2.2 \mathrm{~Hz},[1 \mathrm{H}]$, EDMIM H4/5), 4.07 (q, J= 7.4 Hz, [2H], $\mathrm{NCH}_{2}$ ), $3.74(\mathrm{~s}$, $\left.[3 \mathrm{H}], \mathrm{NCH}_{3}\right), 2.56\left(\mathrm{~s},[3 \mathrm{H}], \mathrm{CCH}_{3}\right), 1.47\left(\mathrm{t}, J=7.4 \mathrm{~Hz}, \mathrm{CH}_{3}\right) \cdot{ }^{13} \mathrm{C}\left\{{ }^{1} \mathrm{H}\right\} \mathrm{NMR}\left(75.4 \mathrm{MHz}, \mathrm{CDCl}_{3}\right): 161.82\left(\mathrm{C}, \mathrm{q}, J_{\mathrm{C}-\mathrm{B}}\right.$ $\left.=50.0 \mathrm{~Hz}, \mathrm{BAr}^{\mathrm{F}} \mathrm{C} 1\right), 134.71\left(\mathrm{CH}, \mathrm{BAr}^{\mathrm{F}} \mathrm{C} 2 / 6\right), 128.77\left(\mathrm{C}, \mathrm{q}, J_{\mathrm{C}-\mathrm{F}}=3.3 \mathrm{~Hz}, \mathrm{BAr}^{\mathrm{F}} \mathrm{C} 3 / 5\right), 124.45\left(\mathrm{C}, \mathrm{q}, J_{C-F}=273\right.$ $\left.\mathrm{Hz}, \mathrm{CF}_{3}\right), 122.40,120.32$ (each $\mathrm{CH}$, EDMIM C4/5), $117.51\left(\mathrm{CH}, \mathrm{BAr}{ }^{\mathrm{F}} \mathrm{C} 4\right), 44.11\left(\mathrm{CH}_{2}\right), 34.94\left(\mathrm{NCH}_{3}\right), 14.37$ $\left(\mathrm{CCH}_{3}\right), 8.87\left(\mathrm{CH}_{3}\right) \mathrm{ppm} .{ }^{19} \mathrm{~F}\left\{{ }^{1} \mathrm{H}\right\} \mathrm{NMR}\left(282.4 \mathrm{MHz}, \mathrm{CDCl}_{3}\right):-62.72 \mathrm{ppm}$.

[HMIM][BArF]: [HMIM]Cl (113 mg, $0.56 \mathrm{mmol}$ ) and $\mathrm{Na}\left[\mathrm{BAr}^{\mathrm{F}}\right.$ ] (500 $\mathrm{mg}, 0.56 \mathrm{mmol}$ ) were suspended in $\mathrm{CH}_{2} \mathrm{Cl}_{2}(20 \mathrm{~mL})$ and stirred for two hours. After filtration through a short plug of silica, the $\mathrm{CH}_{2} \mathrm{Cl}_{2}$ was removed in vacuo, affording $409 \mathrm{mg}$ of a colourless liquid in $71 \%$ yield. This crystallized upon standing for $\sim 16$ hours. Anal. calc. for $\mathrm{C}_{42} \mathrm{H}_{31} \mathrm{~N}_{2} \mathrm{BF}_{24}$ (1030.21): C 48.82; H 3.03; N 2.72. Found C 48.67; H 2.93; N 2.83. ${ }^{1} \mathrm{H}$ NMR (300.1 MHz, $\mathrm{CD}_{2} \mathrm{Cl}_{2}$ ): 8.07 (s, [1H], H2), 7.74 (s, [8H], BAr $\left.{ }^{\mathrm{F}} \mathrm{H} 2 / 6\right), 7.58$ (s, [4H], BArF H4), 7.25, 7.20 (each s, $[1 \mathrm{H}], \mathrm{H} 4 / 5), 4.10\left(\mathrm{q}, J=7.3 \mathrm{~Hz},[2 \mathrm{H}], \mathrm{NCH}_{2}\right), 3.86\left(\mathrm{~s},[3 \mathrm{H}], \mathrm{NCH}_{3}\right), 1.78-1.92\left(\mathrm{~m},[2 \mathrm{H}], \mathrm{CH}_{2}\right), 1.31(\mathrm{~s}$, $\left.[6 \mathrm{H}], \mathrm{CH}_{2}\right), 0.87\left(\mathrm{t}, J=6.2 \mathrm{~Hz},[3 \mathrm{H}], \mathrm{CH}_{3}\right) \mathrm{ppm} .{ }^{13} \mathrm{C}\left\{{ }^{1} \mathrm{H}\right\} \mathrm{NMR}\left(75.5 \mathrm{MHz}, \mathrm{CD}_{2} \mathrm{Cl}_{2}\right): 162.35\left(\mathrm{C}, \mathrm{q}, J_{\mathrm{C}-\mathrm{B}}=50.9 \mathrm{~Hz}\right.$, BAr $^{F}$ C1), $135.37\left(\mathrm{CH}, \mathrm{BAr}^{\mathrm{F}} \mathrm{C} 2 / 6\right), 129.70\left(\mathrm{C}, \mathrm{q}, J_{\mathrm{C}-\mathrm{F}}=3.3 \mathrm{~Hz}, \mathrm{BAr}^{\mathrm{F}} \mathrm{C} 3 / 5\right), 125.19\left(\mathrm{C}, \mathrm{q}, J_{C-F}=272 \mathrm{~Hz}_{\mathrm{C}} \mathrm{CF}_{3}\right)$, 124.65, 123.49 (each $\mathrm{CH}, \mathrm{C} 4 / 5), 118.10\left(\mathrm{CH}, \mathrm{BAr}{ }^{\mathrm{F}} \mathrm{C} 4\right), 51.44\left(\mathrm{NCH}_{2}\right), 37.27\left(\mathrm{NCH}_{3}\right), 31.41,30.46,26.31$, 22.81 (each $\mathrm{CH}_{2}$ ), $14.05\left(\mathrm{CH}_{3}\right)$ ppm. ${ }^{19} \mathrm{~F}\left\{{ }^{1} \mathrm{H}\right\} \mathrm{NMR}\left(282.4 \mathrm{MHz}, \mathrm{CD}_{2} \mathrm{Cl}_{2}\right)$ : -63.10 ppm.

[EMBIM][BAr']: [EMBIM]I (72 mg, $0.25 \mathrm{mmol}$ ) and Na[BAr ${ }^{\mathrm{F}}$ ] $(222 \mathrm{mg}, 0.25 \mathrm{mmol}$ ) were dissolved in acetone $(20 \mathrm{~mL})$ and stirred for four hours. After this time, the resulting colourless solution was concentrated to dryness and extracted into $\mathrm{CH}_{2} \mathrm{Cl}_{2}(2 \times 20 \mathrm{~mL})$. The combined extracts were filtered through a short plug of silica and the $\mathrm{CH}_{2} \mathrm{Cl}_{2}$ was removed in vacuo, affording $190 \mathrm{mg}$ of a white solid in $74 \%$ yield. Crystals were obtained through the slow diffusion of pentane into a concentrated $\mathrm{CH}_{2} \mathrm{Cl}_{2}$ solution. Anal. calc. for $\mathrm{C}_{42} \mathrm{H}_{25} \mathrm{~N}_{2} \mathrm{BF}_{24}$ (1024.62): C 49.23; H 2.46; N 2.73. Found C 49.11; H 2.53; N 2.76. ${ }^{1} \mathrm{H}$ NMR (400.1 MHz, $\left.\left(\mathrm{CD}_{3}\right)_{2} \mathrm{CO}\right): 9.68(\mathrm{~s},[1 \mathrm{H}], \mathrm{H} 2), 8.05-8.14(\mathrm{~m},[2 \mathrm{H}], \mathrm{Ph}), 7.76-7.83\left(\mathrm{~m},[10 \mathrm{H}], \mathrm{BAr}^{\mathrm{F}} \mathrm{H} 2 / 6\right.$ and $\mathrm{Ph}$ ), $7.67\left(\mathrm{~s},[4 \mathrm{H}], \mathrm{BAr}^{\mathrm{F}} \mathrm{H} 4\right), 4.76\left(\mathrm{q}, J=7.3 \mathrm{~Hz},[2 \mathrm{H}], \mathrm{NCH}_{2}\right), 4.32\left(\mathrm{~s},[3 \mathrm{H}], \mathrm{NCH}_{3}\right), 1.71(\mathrm{t}, J=7.3 \mathrm{~Hz}$, [3H], $\left.\mathrm{CH}_{3}\right)$ ppm. (400.1 MHz, $\left.\mathrm{CD}_{2} \mathrm{Cl}_{2}\right): 8.50(\mathrm{~s},[1 \mathrm{H}], \mathrm{H} 2), 7.76-7.81(\mathrm{~m},[4 \mathrm{H}], \mathrm{Ph}), 7.72\left(\mathrm{~s},[8 \mathrm{H}], \mathrm{BAr}^{\mathrm{F}} \mathrm{H} 2 / 6\right)$, 7.55 (s, [4H], BAr ${ }^{\mathrm{F}} \mathrm{H}$ ) , 4.47 (q, J= $\left.7.3 \mathrm{~Hz},[2 \mathrm{H}], \mathrm{NCH}_{2}\right), 4.10\left(\mathrm{~s},[3 \mathrm{H}], \mathrm{NCH}_{3}\right), 1.68\left(\mathrm{t}, J=7.3 \mathrm{~Hz},[3 \mathrm{H}], \mathrm{CH}_{3}\right)$. ${ }^{13} \mathrm{C}\left\{{ }^{1} \mathrm{H}\right\}$ NMR $\left(100.6 \mathrm{MHz},\left(\mathrm{CD}_{3}\right)_{2} \mathrm{CO}\right): 162.70\left(\mathrm{C}, \mathrm{q}, \mathrm{J}_{\mathrm{C}-\mathrm{B}}=50.9 \mathrm{~Hz}, \mathrm{BAr}{ }^{\mathrm{F}} \mathrm{C} 1\right), 142.91(\mathrm{CH}, \mathrm{C} 2), 135.64(\mathrm{CH}$, BAr $^{\mathrm{F}} \mathrm{C} 2 / 6$ ), 133.54, 132.40 (each $\mathrm{CH}, \mathrm{Ph}$ ), 130.11 (C, qq, $\mathrm{J}_{\mathrm{C}-\mathrm{F}}=32.2,3.3 \mathrm{~Hz}, \mathrm{BAr}^{\mathrm{F}} \mathrm{C} 3 / 5$ ), 127.99, 127.94 (each $\mathrm{CH}, \mathrm{Ph}$ ), $125.47\left(\mathrm{C}, \mathrm{q}, J_{\mathrm{C}-\mathrm{F}}=272 \mathrm{~Hz}, \mathrm{CF}_{3}\right.$ ), 124.65, 123.49 (each CH, EMIM C4/5), 118.54 (CH, septet, $\left.{ }^{3} J_{C-F}=4.0 \mathrm{~Hz}, \mathrm{BAr}^{\mathrm{F}} \mathrm{C} 4\right), 43.57\left(\mathrm{NCH}_{2}\right), 34.08\left(\mathrm{NCH}_{3}\right), 14.85\left(\mathrm{CH}_{3}\right)$ ppm. ${ }^{19} \mathrm{~F}\left\{{ }^{1} \mathrm{H}\right\} \mathrm{NMR}\left(282.4 \mathrm{MHz}, \mathrm{CDCl}_{3}\right):-$ $62.65 \mathrm{ppm}$.

[IDiPPH][BAr ${ }^{\mathrm{F}}$ ]: [IDiPPH]Cl (106 mg, $\left.0.25 \mathrm{mmol}\right)$ and $\mathrm{Na}\left[\mathrm{BAr}^{\mathrm{F}}\right]$ (222 mg, $\left.0.25 \mathrm{mmol}\right)$ were dissolved in acetone $(20 \mathrm{~mL})$ and stirred for four hours. After this time, the resulting colourless solution was concentrated to dryness and extracted into $\mathrm{CH}_{2} \mathrm{Cl}_{2}(2 \times 20 \mathrm{~mL})$. The combined extracts were filtered through a short plug of silica and the $\mathrm{CH}_{2} \mathrm{Cl}_{2}$ was removed in vacuo, affording $224 \mathrm{mg}$ of a white solid in $72 \%$ yield. Crystals were obtained through the slow diffusion of pentane into a concentrated $\mathrm{CH}_{2} \mathrm{Cl}_{2}$ solution. Anal. calc. for $\mathrm{C}_{59} \mathrm{H}_{49} \mathrm{~N}_{2} \mathrm{BF}_{24}$ (1253.00): $\mathrm{C} 56.56 ; \mathrm{H} 3.94 ; \mathrm{N} 2.24$. Found $\mathrm{C} 56.43 ; \mathrm{H} 4.05 ; \mathrm{N} 2.31 .{ }^{1} \mathrm{H}$ NMR (400.1 MHz, $\left(\mathrm{CDCl}_{3}\right): 8.20$ (s, [1H], IDiPPH H2), 7.68 (br s, [8H], BAr $\left.{ }^{\mathrm{F}} \mathrm{H} 2 / 6\right), 7.63(\mathrm{t}, J=8.1 \mathrm{~Hz},[2 \mathrm{H}]$, IDiPPH Ar), 7.53 (d, J = $1.7 \mathrm{~Hz},[2 \mathrm{H}]$, IDiPPH H4/5), 7.50 (br s, [4H], BAr $\left.{ }^{\mathrm{F}} \mathrm{H} 4\right), 7.39$ (d, J = $7.8 \mathrm{~Hz},[4 \mathrm{H}]$, IDiPPH Ar), 2.32 (septet, $J=6.8 \mathrm{~Hz},{ }^{i} \mathrm{Pr} \mathrm{CH}$ ), 1.25, 1.17 (each d, $\left.J=6.8 \mathrm{~Hz},[12 \mathrm{H}],{ }^{i} \operatorname{Pr} \mathrm{CH}_{3}\right) \mathrm{ppm} .(400.1 \mathrm{MHz}$, 
$\left.\mathrm{CD}_{2} \mathrm{Cl}_{2}\right): 8.33\left(\mathrm{~s},[1 \mathrm{H}]\right.$, IDiPPH H2), $7.72\left(\mathrm{br} \mathrm{s},[8 \mathrm{H}], \mathrm{BAr}^{\mathrm{F}} \mathrm{H} 2 / 6\right), 7.63-7.76(\mathrm{~m},[4 \mathrm{H}]$, IDiPPH Ar and IDiPPH $\mathrm{H} 4 / 5$ ), 7.56 (br s, [4H], BArF H4), 7.43 (d, $J=7.8 \mathrm{~Hz},[4 \mathrm{H}]$, IDiPPH Ar), 2.36 (septet, $J=6.8 \mathrm{~Hz},{ }^{\mathrm{P} P \mathrm{CH}}$ ), 1.29, 1.21 (each d, J = 6.8 Hz, [12H], $\left.\operatorname{Pr~CH}_{3}\right)$ ppm. ${ }^{13}{ }^{1}\left\{{ }^{1} \mathrm{H}\right\} \mathrm{NMR}\left(100.6 \mathrm{MHz},\left(\mathrm{CDCl}_{3}\right): 161.62\left(\mathrm{C}, \mathrm{q}, \mathrm{J}_{\mathrm{C}-\mathrm{B}}=50.9 \mathrm{~Hz}\right.\right.$, BArF C1), 144.68 (CH, IDiPPH C2), 136.31 (C, IDiPPH Ar), 134.76 (CH, BArF C2/6), 133.19 (CH, IDiPPH Ar), 128.80 (C, qq, JCF $\left.=32.2,3.3 \mathrm{~Hz}, \mathrm{BAr}^{\mathrm{F}} \mathrm{C} 3 / 5\right), 128.65$ (CH, IDiPPH Ar), 125.86 (C, IDiPPH Ar), 125.28 (CH, IDiPPH C4/5), $124.51\left(\mathrm{C}, \mathrm{q}, J_{\mathrm{C}-\mathrm{F}}=272 \mathrm{~Hz}, \mathrm{CF}_{3}\right), 117.43\left(\mathrm{CH}\right.$, septet, $\left.{ }^{3} \mathrm{~J}_{\mathrm{C}-\mathrm{F}}=4.0 \mathrm{~Hz}, \mathrm{BAr}{ }^{\mathrm{F}} \mathrm{C} 4\right), 29.26(\mathrm{CH})$, 24.42, $23.50\left(\mathrm{CH}_{3}\right)$ ppm. ${ }^{19} \mathrm{~F}\left\{{ }^{1} \mathrm{H}\right\}$ NMR $\left(282.4 \mathrm{MHz}, \mathrm{CDCl}_{3}\right):-62.65 \mathrm{ppm}$.

[BMPYRR][BAr ${ }^{\mathrm{F}}$ ]: [BMPYRR]Br (125 mg, $\left.0.56 \mathrm{mmol}\right)$ and $\mathrm{Na}\left[\mathrm{BAr} \mathrm{F}^{\mathrm{F}}\right.$ ] (500 mg, $0.56 \mathrm{mmol}$ ) were suspended in acetone $(20 \mathrm{~mL})$ and stirred for four hours. The resulting pale yellow solution was filtered through Celite ${ }^{\circledR}$ and volatiles were removed in vacuo. The resulting off-white solid was dissolved in $\mathrm{CH}_{2} \mathrm{Cl}_{2}$ and filtered through a short plug of silica. Crystallization occurred through the vapour diffusion of $\mathrm{Et}_{2} \mathrm{O}$ into a concentrated $\mathrm{CH}_{2} \mathrm{Cl}_{2}$ solution: yield $457 \mathrm{mg}$, 81\%. Anal. calc. for $\mathrm{C}_{41} \mathrm{H}_{32} \mathrm{NBF}_{24}$ (1005.67): C 48.98; $\mathrm{H} 3.21 ; \mathrm{N}$ 1.39. Found C 48.83; H 3.25; N 1.46. ${ }^{1} \mathrm{H}$ NMR $\left(400.1 \mathrm{MHz}, \mathrm{CDCl}_{3}\right)$ : 7.70 (s, [8H], BArF H2/6), 7.55 (s, [4H], BArF H4), 3.25-3.31 (m, [4H], BMPYRR H2/5), 3.09-3.15 (m, [2H], $\left.{ }^{n} \mathrm{Bu} \mathrm{NCH}_{2}\right), 2.84\left(\mathrm{~s},[3 \mathrm{H}], \mathrm{NCH}_{3}\right), 2.13(\mathrm{v}$ br s, [4H], BMPYRR H3/4), 1.60-1.69 (m, [2H], $\left.{ }^{n} \mathrm{Bu} \mathrm{NCH}_{2} \mathrm{CH}_{2}\right), 1.33$ (dq, $\left.J=14.8,7.5 \mathrm{~Hz},[2 \mathrm{H}],{ }^{n} \mathrm{Bu} \mathrm{C}_{2} \mathrm{CH}_{3}\right)$, $\left.0.92\left(\mathrm{t}, J=7.3 \mathrm{~Hz},[3 \mathrm{H}],{ }^{n} \mathrm{Bu} \mathrm{CH}\right) \mathrm{ppm} .{ }^{13} \mathrm{C}^{1}{ }^{1} \mathrm{H}\right\} \mathrm{NMR}\left(100.6 \mathrm{MHz}, \mathrm{CDCl}_{3}\right): 161.65$ (C, q, JC-B $=50.3 \mathrm{~Hz}, \mathrm{BAr}{ }^{\mathrm{F}}$ C1), $134.72\left(\mathrm{CH}, \mathrm{BAr} \mathrm{F}^{\mathrm{C}} \mathrm{C} / 6\right), 129.11$ (C, q, JCF $=2.9 \mathrm{~Hz}, \mathrm{BArF} \mathrm{C} 3$ ), $124.50\left(\mathrm{C}, \mathrm{q}, J_{C-F}=273 \mathrm{~Hz}, \mathrm{CF}_{3}\right), 117.53$ ( $\left.\mathrm{CH}, \mathrm{BAr}{ }^{\mathrm{F}} \mathrm{C} 4\right), 65.27,64.94$ (each $\mathrm{CH}_{2}$, BMPYRR C2/5 and $\left.\mathrm{NCH}_{2}\right), 48.70\left(\mathrm{CH}_{3}, \mathrm{NCH}_{3}\right), 25.60\left(\mathrm{CH}_{2},{ }^{n} \mathrm{Bu} \mathrm{CH}_{2}\right)$, 21.47, $19.45\left(\mathrm{CH}_{2}\right.$, BMPYRR C3/4 and $\left.{ }^{n} \mathrm{Bu} \mathrm{CH}_{2} \mathrm{Me}\right), 13.06\left(\mathrm{CH}_{3},{ }^{n} \mathrm{Bu} \mathrm{CH}_{3}\right) \mathrm{ppm} .{ }^{19} \mathrm{~F}\left\{{ }^{1} \mathrm{H}\right\} \mathrm{NMR}(282.4 \mathrm{MHz}$, $\left.\mathrm{CDCl}_{3}\right):-62.59 \mathrm{ppm}$.

[EMIM][AI( $\left(\mathrm{O}^{+} \mathrm{C}_{4} \mathrm{~F}_{9}\right)_{4}$ ]: [EMIM]Br $(108 \mathrm{mg}, 0.56 \mathrm{mmol})$ and $\mathrm{Li}\left[\mathrm{Al}\left(\mathrm{O}^{\mathrm{t}} \mathrm{C}_{4} \mathrm{~F}_{9}\right)_{4}\right](545 \mathrm{mg}, 0.56 \mathrm{mmol})$ were suspended in acetone $(20 \mathrm{~mL})$ and stirred for four hours. After filtration through Celite ${ }^{\circledast}$, the volatiles were removed in vacuo. The resulting off-white solid was dissolved in $\mathrm{CH}_{2} \mathrm{Cl}_{2}$ and filtered through a short plug of silica. Crystallization occurred from vapour diffusion of $\mathrm{Et}_{2} \mathrm{O}$ into a concentrated $\mathrm{CH}_{2} \mathrm{Cl}_{2}$ solution. Yield: $416 \mathrm{mg}, 69 \%$. Anal. calc. for $\mathrm{C}_{22} \mathrm{H}_{11} \mathrm{~N}_{2} \mathrm{O}_{4} \mathrm{AlF}_{36}$ (1078.00): $\mathrm{C} 24.49 ; \mathrm{H} 1.03 ; \mathrm{N} 2.60$. Found C 24.58; $\mathrm{H}$ 0.90; N 2.66. ${ }^{1} \mathrm{H}$ NMR (300.1 MHz, $\left.\left(\mathrm{CD}_{3}\right)_{2} \mathrm{CO}\right): 9.11(\mathrm{~s},[1 \mathrm{H}], \mathrm{H} 2), 7.82,7.75$ (each s, [1H], H4/5), 4.44 (q, $J=$ $\left.7.3 \mathrm{~Hz},[2 \mathrm{H}], \mathrm{CH}_{2}\right), 4.09\left(\mathrm{~s},[3 \mathrm{H}], \mathrm{NCH}_{3}\right), 1.58\left(\mathrm{t}, J=7.3 \mathrm{~Hz},[3 \mathrm{H}], \mathrm{CH}_{3}\right) \mathrm{ppm} .\left(400.1 \mathrm{MHz}, \mathrm{CD}_{2} \mathrm{Cl}_{2}\right): 8.15(\mathrm{~s}$, $[1 \mathrm{H}], \mathrm{H} 2), 7.35,7.32$ (each s, [1H], H4/5), 4.24 (q, $J=7.4 \mathrm{~Hz},[2 \mathrm{H}], \mathrm{NCH}_{2}$ ), 3.95 (s, [3H], NCH $), 1.61$ (t, $J=$ $\left.7.4 \mathrm{~Hz},[3 \mathrm{H}], \mathrm{CH}_{3}\right) .{ }^{13} \mathrm{C}\left\{{ }^{1} \mathrm{H}\right\} \mathrm{NMR}\left(75.5 \mathrm{MHz},\left(\mathrm{CD}_{3}\right)_{2} \mathrm{CO}\right): 125.34,123.71(\mathrm{CH}, \mathrm{C} 4 / 5), 122.67$ (q, Jc-F $=294.8 \mathrm{~Hz}$, $\left.\mathrm{CF}_{3}\right), 46.32\left(\mathrm{CH}_{2}\right), 37.16\left(\mathrm{NCH}_{3}\right), 16.11\left(\mathrm{CH}_{3}\right) \mathrm{ppm} .{ }^{19} \mathrm{~F}\left\{{ }^{1} \mathrm{H}\right\} \mathrm{NMR}\left(282.4 \mathrm{MHz},\left(\mathrm{CD}_{3}\right)_{2} \mathrm{CO}\right):-75.12 \mathrm{ppm}$.

[EDMIM][Al( $\left.\left(\mathrm{O}^{t} \mathrm{C}_{4} \mathrm{~F}_{9}\right)_{4}\right]$ : [EDMIM]Cl $(90 \mathrm{mg}, 0.56 \mathrm{mmol})$ and $\mathrm{Li}\left[\mathrm{Al}\left(\mathrm{O}^{\mathrm{t}} \mathrm{C}_{4} \mathrm{~F}_{9}\right)_{4}\right](545 \mathrm{mg}, 0.56 \mathrm{mmol})$ were suspended in acetone $(20 \mathrm{~mL})$ and stirred for four hours. After filtration through Celite ${ }^{\circledast}$, the volatiles were removed in vacuo. The resulting sticky solid was dissolved in $\mathrm{CH}_{2} \mathrm{Cl}_{2}(\sim 50 \mathrm{~mL})$ and filtered through a short plug of silica. Volatiles were removed in vacuo, affording a very pale green solid. Yield: $416 \mathrm{mg}, 68 \%$. Crystallization occurred from slow evaporation of the $\mathrm{CH}_{2} \mathrm{Cl}_{2}$ solution. Anal. calc. for $\mathrm{C}_{23} \mathrm{H}_{13} \mathrm{~N}_{2} \mathrm{O}_{4} \mathrm{AlF}_{36}$ (1092.01): C 25.27; H 1.20; N 2.56. Found C 25.36; H 1.13; N 2.55. ${ }^{1} \mathrm{H}$ NMR $\left(300.0 \mathrm{MHz},\left(\mathrm{CD}_{3}\right)_{2} \mathrm{CO}\right): 7.68$, 7.63 (each d, $J=2.2 \mathrm{~Hz},[2 \mathrm{H}], \mathrm{H} 4 / 5), 4.36\left(\mathrm{q}, J=7.1 \mathrm{~Hz},[2 \mathrm{H}], \mathrm{CH}_{2}\right), 3.98\left(\mathrm{~s},[3 \mathrm{H}], \mathrm{NCH}_{3}\right), 2.82\left(\mathrm{~s},[3 \mathrm{H}], \mathrm{CCH}_{3}\right)$, $1.50\left(\mathrm{t}, J=7.3 \mathrm{~Hz},[3 \mathrm{H}], \mathrm{CH}_{3}\right)$ ppm. $\left(400.1 \mathrm{MHz}, \mathrm{CD}_{2} \mathrm{Cl}_{2}\right): 7.22,7.19$ (each d, $\left.J=2.2 \mathrm{~Hz},[1 \mathrm{H}], \mathrm{H} 4 / 5\right), 4.12$ (q, $\left.J=7.4 \mathrm{~Hz},[2 \mathrm{H}], \mathrm{NCH}_{2}\right), 3.80\left(\mathrm{~s},[3 \mathrm{H}], \mathrm{NCH}_{3}\right), 2.61\left(\mathrm{~s},[3 \mathrm{H}], \mathrm{CCH}_{3}\right), 1.52\left(\mathrm{t}, J=7.4 \mathrm{~Hz},[3 \mathrm{H}], \mathrm{CH}_{3}\right) .{ }^{13} \mathrm{C}\left\{{ }^{1} \mathrm{H}\right\} \mathrm{NMR}$ (75.4 MHz, $\left.\left(\mathrm{CD}_{3}\right)_{2} \mathrm{CO}\right): 124.03(\mathrm{CH}), 122.66\left(\mathrm{q}, \mathrm{J}_{\mathrm{C}-\mathrm{F}}=291.8 \mathrm{~Hz}, \mathrm{CF}_{3}\right), 121.89(\mathrm{CH}), 44.84\left(\mathrm{CH}_{2}\right), 35.99\left(\mathrm{NCH}_{3}\right)$, $15.74\left(\mathrm{CCH}_{3}\right), 10.09\left(\mathrm{CH}_{3}\right)$ ppm. ${ }^{19} \mathrm{~F}\left\{{ }^{1} \mathrm{H}\right\} \mathrm{NMR}\left(282.4 \mathrm{MHz},\left(\mathrm{CD}_{3}\right)_{2} \mathrm{CO}\right):-75.12 \mathrm{ppm}$.

[HMIM] [AI( $\left.\left(\mathrm{O}^{+} \mathrm{C}_{4} \mathrm{~F}_{9}\right)_{4}\right]:$ : $\left.\mathrm{HMIM}\right] \mathrm{Cl}(113 \mathrm{mg}, 0.56 \mathrm{mmol})$ and $\mathrm{Li}\left[\mathrm{Al}\left(\mathrm{O}^{+} \mathrm{C}_{4} \mathrm{~F}_{9}\right)_{4}\right](545 \mathrm{mg}, 0.56 \mathrm{mmol})$ were suspended in $\mathrm{CH}_{2} \mathrm{Cl}_{2}(20 \mathrm{~mL})$ and stirred for two hours. After filtration through a short plug of silica, the $\mathrm{CH}_{2} \mathrm{Cl}_{2}$ was removed in vacuo. Yield: $496 \mathrm{mg}$ (78\% yield). Crystallization occurred through the vapour 
diffusion of pentane into a concentrated $\mathrm{CH}_{2} \mathrm{Cl}_{2}$ solution at $-18{ }^{\circ} \mathrm{C}$. Anal. calc. for $\mathrm{C}_{26} \mathrm{H}_{19} \mathrm{~N}_{2} \mathrm{O}_{4} \mathrm{AlF}_{36}$ (1134.34): C 27.53; H 1.69; N 2.47. Found C 27.65; H 1.75; N 2.54. ${ }^{1} \mathrm{H}$ NMR $\left(300.1 \mathrm{MHz}, \mathrm{CD}_{2} \mathrm{Cl}_{2}\right): 8.11$ (s, [1H], H2), 7.31, 7.30 (each s, [1H], H4/5), 4.14 (t, J= $7.5 \mathrm{~Hz},[2 \mathrm{H}], \mathrm{NCH}_{2}$ ), $3.94\left(\mathrm{~s},[3 \mathrm{H}], \mathrm{NCH}_{3}\right.$ ), 1.89 (septet, $\left.J=7.1 \mathrm{~Hz},[2 \mathrm{H}], \mathrm{NCH}_{2} \underline{\mathrm{C}}_{2}\right), 1.34\left(\mathrm{br} \mathrm{s},[6 \mathrm{H}], \mathrm{CH}_{2}\right), 0.90\left(\mathrm{t}, J=7.0 \mathrm{~Hz},[3 \mathrm{H}], \mathrm{CH}_{3}\right) \mathrm{ppm} .{ }^{13} \mathrm{C}\left\{{ }^{1} \mathrm{H}\right\} \mathrm{NMR}(75.5$ $\mathrm{MHz}, \mathrm{CD}_{2} \mathrm{Cl}_{2}$ ): 125.34, $123.71(\mathrm{CH}, \mathrm{C} 4 / 5), 122.67$ (q, J $\left.\mathrm{J}_{\mathrm{-F}}=294.8 \mathrm{~Hz}, \mathrm{CF}_{3}\right), 51.47\left(\mathrm{NCH}_{2}\right), 37.31\left(\mathrm{NCH}_{3}\right)$, 31.44, 30.47, 26.34, $22.84\left(\mathrm{CH}_{2}\right), 14.07\left(\mathrm{CH}_{3}\right) \mathrm{ppm} .{ }^{19} \mathrm{~F}\left\{{ }^{1} \mathrm{H}\right\} \mathrm{NMR}\left(282.4 \mathrm{MHz}, \mathrm{CD}_{2} \mathrm{Cl}_{2}\right):-76.00 \mathrm{ppm}$.

[EMBIM][Al( $\left(\mathrm{O}^{t} \mathrm{C}_{4} \mathrm{~F}_{9}\right)_{4}$ ]: [EMBIM]I $(72 \mathrm{mg}, 0.25 \mathrm{mmol})$ and $\mathrm{Li}\left[\mathrm{Al}\left(\mathrm{O}^{\mathrm{t}} \mathrm{C}_{4} \mathrm{~F}_{9}\right)_{4}\right.$ ] (243 $\left.\mathrm{mg}, 0.25 \mathrm{mmol}\right)$ were dissolved in $\mathrm{CH}_{2} \mathrm{Cl}_{2}(20 \mathrm{~mL})$ and stirred for four hours. After filtration through a short plug of silica, the $\mathrm{CH}_{2} \mathrm{Cl}_{2}$ was removed in vacuo. Yield: $197 \mathrm{mg}, 70 \%$. Anal. calc. for $\mathrm{C}_{26} \mathrm{H}_{13} \mathrm{~N}_{2} \mathrm{O}_{4} \mathrm{AlF}_{36}$ (1128.30): C 27.68; $\mathrm{H}$ 1.16; N 2.48. Found C 27.81; H 1.04; N 2.39. ${ }^{1} \mathrm{H}$ NMR (400.1 MHz, $\left.\left(\mathrm{CD}_{3}\right)_{2} \mathrm{CO}\right): 9.68$ (s, [1H], H2), 8.05-8.15 $(\mathrm{m},[2 \mathrm{H}], \mathrm{Ph}), 7.76-7.81(\mathrm{~m},[2 \mathrm{H}], \mathrm{Ph}), 4.76\left(\mathrm{q}, J=7.3 \mathrm{~Hz},[2 \mathrm{H}], \mathrm{NCH}_{2}\right), 4.32\left(\mathrm{~s},[3 \mathrm{H}], \mathrm{NCH}_{3}\right), 1.71(\mathrm{t}, J=7.3$ $\left.\mathrm{Hz},[3 \mathrm{H}], \mathrm{CH}_{3}\right)$ ppm. (400.1 MHz, $\left.\mathrm{CD}_{2} \mathrm{Cl}_{2}\right): 8.54(\mathrm{~s},[1 \mathrm{H}], \mathrm{H} 2), 7.79-7.85(\mathrm{~m},[4 \mathrm{H}], \mathrm{Ph}), 4.51(\mathrm{q}, J=7.3 \mathrm{~Hz}$, $\left.\mathrm{NCH}_{2}\right), 4.14\left(\mathrm{~s},[3 \mathrm{H}], \mathrm{NCH}_{3}\right), 1.72\left(\mathrm{t}, J=7.3 \mathrm{~Hz}, \mathrm{CH}_{3}\right) .{ }^{13} \mathrm{C}\left\{{ }^{1} \mathrm{H}\right\} \mathrm{NMR}\left(100.6 \mathrm{MHz},\left(\mathrm{CD}_{3}\right)_{2} \mathrm{CO}\right): 143.22(\mathrm{CH})$, 133.86, $132.71(\mathrm{CH}, \mathrm{Ph}), 130.98(\mathrm{C}, \mathrm{Ph}), 122.56$ (q, J $\left.J_{\mathrm{C}-\mathrm{F}}=291 \mathrm{~Hz}, \mathrm{CF}_{3}\right), 43.88\left(\mathrm{CH}_{2}\right), 34.39\left(\mathrm{NCH}_{3}\right), 15.15$ $\left(\mathrm{CH}_{3}\right)$ ppm. ${ }^{19} \mathrm{~F}\left\{{ }^{1} \mathrm{H}\right\}$ NMR (282.4 MHz, $\left.\left(\mathrm{CD}_{3}\right)_{2} \mathrm{CO}\right):-75.08 \mathrm{ppm}$. 


\begin{tabular}{|c|c|c|c|c|}
\hline Compound & [EMIM][BArF] & [EDMIM][BArF] & [HMIM][BArF] & [BMPYRR][BArF] \\
\hline Formula & $\mathrm{C}_{38} \mathrm{H}_{23} \mathrm{BF}_{24} \mathrm{~N}_{2}$ & $\mathrm{C}_{39} \mathrm{H}_{25} \mathrm{BF}_{24} \mathrm{~N}_{2}$ & $\mathrm{C}_{42} \mathrm{H}_{31} \mathrm{BF}_{24} \mathrm{~N}_{2}$ & $\mathrm{C}_{41} \mathrm{H}_{32} \mathrm{BF}_{24} \mathrm{~N}$ \\
\hline $\mathrm{M} / \mathrm{g} \mathrm{mol}^{-1}$ & 974.39 & 988.42 & 1030.50 & 1005.49 \\
\hline Crystal system & monoclinic & monoclinic & monoclinic & triclinic \\
\hline Space group (No.) & $C 2 / c(15)$ & C 2/c (15) & $P 2_{1} / c(14)$ & $P-1(2)$ \\
\hline$a / \AA ̊$ & $23.112(6)$ & $23.113(15)$ & $18.901(9)$ & $16.463(1)$ \\
\hline$b / \AA ̊$ & $9.100(2)$ & $9.231(6)$ & $13.222(7)$ & $17.586(1)$ \\
\hline$c / \AA ̊$ & $19.022(5)$ & $19.075(12)$ & $16.877(9)$ & $18.228(1)$ \\
\hline$\alpha /^{\circ}$ & 90 & 90 & 90 & $110.823(8)$ \\
\hline$\beta /^{\circ}$ & $99.852(5)$ & $100.128(11)$ & $97.13(1)$ & $103.281(7)$ \\
\hline$\gamma /{ }^{\circ}$ & 90 & 90 & 90 & $110.957(8)$ \\
\hline$U / \AA^{3}$ & 3941.7(17) & $4006(4)$ & $4185(4)$ & $4203.9(8)$ \\
\hline Z & 4 & 4 & 4 & 4 \\
\hline$\mu(\mathrm{Mo}-\mathrm{K} \alpha) / \mathrm{mm}^{-1}$ & 0.176 & 0.174 & 0.171 & 1.589 \\
\hline$F(000)$ & 1944 & 1976 & 2072 & 2024 \\
\hline Total reflections & 9972 & 8298 & 25672 & 44339 \\
\hline Unique reflections & 4018 & 3522 & 9518 & 19234 \\
\hline$R_{\text {int }}$ & 0.083 & 0.050 & 0.054 & 0.063 \\
\hline $\begin{array}{l}\text { Goodness-of-fit on } \\
F^{2}\end{array}$ & 1.043 & 1.171 & 1.128 & 1.026 \\
\hline$R_{1}^{\mathrm{b}}\left[I_{\circ}>2 \sigma\left(I_{\circ}\right)\right]$ & 0.077 & 0.088 & 0.083 & 0.082 \\
\hline$R_{1}$ (all data) & 0.086 & 0.123 & 0.119 & 0.161 \\
\hline$w R_{2}^{\mathrm{b}}\left[I_{o}>2 \sigma\left(I_{0}\right)\right]$ & 0.206 & 0.136 & 0.158 & 0.162 \\
\hline$w R_{2}$ (all data) & 0.214 & 0.152 & 0.180 & 0.195 \\
\hline $\begin{array}{l}\text { Largest diff. peak } \\
\text { and hole }\left(\mathrm{e}^{-3}\right)\end{array}$ & $1.027,-0.918$ & $0.551,-0.259$ & $0.389,-0.410$ & $0.931,-0.718$ \\
\hline
\end{tabular}




\begin{tabular}{|c|c|c|c|c|}
\hline Compound & $\begin{array}{l}{[E M B I M]\left[B A r^{F}\right] \cdot C} \\
\mathrm{H}_{2} \mathrm{Cl}_{2}\end{array}$ & [IDiPPH][BArF] & $\begin{array}{l}{[E M I M]\left[A I\left(O^{t} C_{4} F_{9}\right)\right.} \\
\text { 4] }\end{array}$ & $\begin{array}{l}\text { [EDMIM] [AI }\left(\mathrm{O}^{t} \mathrm{C}_{4} \mathrm{~F}\right. \\
\left.\left.{ }_{9}\right)_{4}\right]\end{array}$ \\
\hline Formula & $\begin{array}{l}\mathrm{C}_{42} \mathrm{H}_{25} \mathrm{BF}_{24} \mathrm{~N}_{2} \cdot \mathrm{C} \\
\mathrm{H}_{2} \mathrm{Cl}_{2}\end{array}$ & $\mathrm{C}_{59} \mathrm{H}_{49} \mathrm{BF}_{24} \mathrm{~N}_{2}$ & $\mathrm{C}_{22} \mathrm{H}_{11} \mathrm{AlF}_{36} \mathrm{~N}_{2} \mathrm{O}_{4}$ & $\mathrm{C}_{23} \mathrm{H}_{13} \mathrm{AlF}_{36} \mathrm{~N}_{2} \mathrm{O}_{4}$ \\
\hline $\mathrm{M} / \mathrm{g} \mathrm{mol}^{-1}$ & 1109.37 & 1252.81 & 1078.31 & 1092.33 \\
\hline Crystal system & triclinic & monoclinic & monoclinic & triclinic \\
\hline Space group (No.) & $P-1(2)$ & $P 2_{1} / n(14)$ & $P 2 \sqrt{1} / c(14)$ & $P-1(2)$ \\
\hline$a / \AA ̊$ & $12.2647(5)$ & $18.685(3)$ & $11.724(4)$ & $9.544(5)$ \\
\hline$b / \AA ̊$ & $13.6343(5)$ & $17.676(2)$ & $15.811(5)$ & $12.396(7)$ \\
\hline$c / \AA ̊$ & $14.9811(5)$ & $19.680(3)$ & $18.680(6)$ & 15.641(9) \\
\hline$\alpha /^{\circ}$ & $76.854(3)$ & 90 & 90 & $88.23(2)$ \\
\hline$\beta /^{\circ}$ & $75.421(3)$ & $118.318(2)$ & $99.958(5)$ & $82.22(2)$ \\
\hline$\gamma /{ }^{\circ}$ & $67.846(4)$ & 90 & 90 & $78.05(2)$ \\
\hline$U / \AA^{3}$ & $2220.82(16)$ & 5722.1(15) & $3410(2)$ & $1794(2)$ \\
\hline$Z$ & 2 & 4 & 4 & 2 \\
\hline$\mu(\mathrm{Mo}-\mathrm{K} \alpha) / \mathrm{mm}^{-1}$ & 0.284 & 0.139 & 0.295 & 0.282 \\
\hline$F(000)$ & 1108 & 2552 & 2104 & 1068 \\
\hline Total reflections & 34008 & 36828 & 17375 & 19518 \\
\hline Unique reflections & 10153 & 11675 & 6002 & 10319 \\
\hline$R_{\text {int }}$ & 0.035 & 0.063 & 0.041 & 0.035 \\
\hline Goodness-of-fit on $F^{2}$ & 1.019 & 0.988 & 1.201 & 1.155 \\
\hline$R_{1}^{\mathrm{b}}\left[I_{0}>2 \sigma\left(I_{0}\right)\right]$ & 0.073 & 0.045 & 0.055 & 0.069 \\
\hline$R_{1}$ (all data) & 0.091 & 0.071 & 0.064 & 0.102 \\
\hline$w R_{2}^{\mathrm{b}}\left[I_{o}>2 \sigma\left(I_{0}\right)\right]$ & 0.202 & 0.110 & 0.100 & 0.126 \\
\hline$w R_{2}$ (all data) & 0.219 & 0.121 & 0.104 & 0.143 \\
\hline $\begin{array}{l}\text { Largest diff. peak and } \\
\text { hole }\left(\mathrm{e}^{-3}\right)\end{array}$ & $1.440,-1.065$ & $0.580,-0.447$ & $0.298,-0.312$ & $0.596,-0.349$ \\
\hline
\end{tabular}




\section{Results and discussion}

\section{Synthesis and solution phase analysis:}

Synthesis of the ionic liquids was carried out in air by a series of salt metathesis reactions between the relevant imidazolium halide and alkali metal salt of the WCA (Scheme 1).

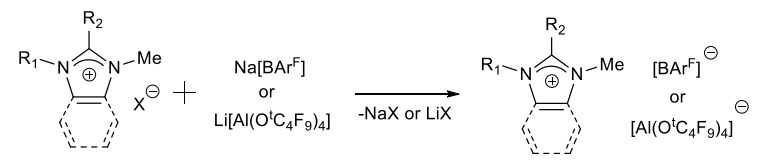

Scheme 1: synthesis of the ionic liquids reported in this paper. $\mathrm{R}_{1}=\mathrm{Et}, \mathrm{n}$-hexyl; $\mathrm{R}_{2}=\mathrm{H}, \mathrm{Me} ; \mathrm{X}=\mathrm{Cl}, \mathrm{Br}$.

All of the reported salts were isolated as white crystalline solids, although the $\left[\mathrm{Al}\left(\mathrm{O}^{t} \mathrm{C}_{4} \mathrm{~F}_{9}\right)_{4}\right]^{-}$salts were mildly hygroscopic and became slightly coloured over time, which necessitated long-term storage under an inert atmosphere. NMR spectroscopy $\left({ }^{1} \mathrm{H},{ }^{19} \mathrm{~F}\left\{{ }^{1} \mathrm{H}\right\}\right)$ was consistent with the presence of 'free' ions in solution with negligible $(\sim 0.05 \mathrm{ppm})$ differences observed between $\left[\mathrm{BAr}{ }^{\mathrm{F}}\right]^{-}$and $\left[\mathrm{Al}\left(\mathrm{O}^{\mathrm{t}} \mathrm{C}_{4} \mathrm{~F}_{9}\right)_{4}\right]^{-}$salts of the same cation (Table 2). The chemical shifts of the $\mathrm{H} 2$ and $\mathrm{H} 4 / 5$ protons have previously been used to indicate the degree of hydrogen bonding in solution between imidazolium cations and $\left[\mathrm{GeX}_{3}\right]^{-}$or $\left[\mathrm{LnX}_{6}\right]^{3-}$ anions $(\mathrm{X}=\mathrm{Cl}, \mathrm{Br}, \mathrm{I} ; \mathrm{Ln}=\mathrm{Sc}, \mathrm{Y}, \mathrm{La}, \mathrm{Ce})$. Significant high-frequency shifts were observed when the NMR spectra of halometallate salts were compared to those containing $\left[\mathrm{BF}_{4}\right]^{-}$or $\left[\mathrm{PF}_{6}\right]^{-12,22}$

\begin{tabular}{|l|l|l|l|l|}
\hline & \multicolumn{2}{|c|}{$\left[^{-1} \mathrm{BAr}\right]^{-}$} & \multicolumn{2}{c|}{$\left[\mathrm{Al}\left(\mathrm{OC}_{4} \mathrm{~F}_{9}\right)_{4}\right]^{-}$} \\
\hline & \multicolumn{1}{|c|}{$\mathrm{H} 2$} & \multicolumn{1}{c|}{$\mathrm{H} 4 / 5$} & \multicolumn{1}{c|}{$\mathrm{H} / 5$} \\
\hline EMIM & 8.10 & $7.25,7.29$ & 8.15 & $7.32,7.35$ \\
\hline HMIM & 8.07 & $7.25,7.20$ & 8.11 & $7.31,7.30$ \\
\hline EMBIM & 8.50 & $\mathrm{n} / \mathrm{a}$ & 8.54 & $\mathrm{n} / \mathrm{a}$ \\
\hline EDMIM & n/a & $7.12,7.16$ & $\mathrm{n} / \mathrm{a}$ & $7.22,7.19$ \\
\hline
\end{tabular}

Table 2: selected solution NMR data for the imidazolium-based cations reported in this paper. All spectra were recorded at $5.0 \mathrm{mM}$ concentration in $\mathrm{CD}_{2} \mathrm{Cl}_{2}$. Chemical shifts quoted in ppm.

The ${ }^{1} \mathrm{H}$ NMR data for the salts synthesized in this study did not show a high-frequency shift of the signals associated with the $\mathrm{H} 2$ and $\mathrm{H} 4 / 5$ protons. The signals were also sharp and well resolved, indicating that solution-phase hydrogen bonding between the imidazolium cations and WCAs was negligible. As a point of comparison, the ${ }^{1} \mathrm{H}$ NMR spectrum of [EMIM]Cl $\left(5.0 \mathrm{mM}\right.$ in $\left.\mathrm{CD}_{2} \mathrm{Cl}_{2}\right)$ has a very broad singlet at 11.17 ppm which is indicative of significant hydrogen bonding in solution.

\section{Solid phase analysis:}

In order to determine whether any interactions were present in the solid state, single crystal X-ray diffraction was employed. Single crystals of [EMIM][BArF], [HMIM][BAr $\left.{ }^{\mathrm{F}}\right]$, [EDMIM][BAr $\left.{ }^{\mathrm{F}}\right]$ and [EMBIM] [BAr $\left.{ }^{\mathrm{F}}\right]$ were obtained and their solid state structures were determined (Figures 2-5). 


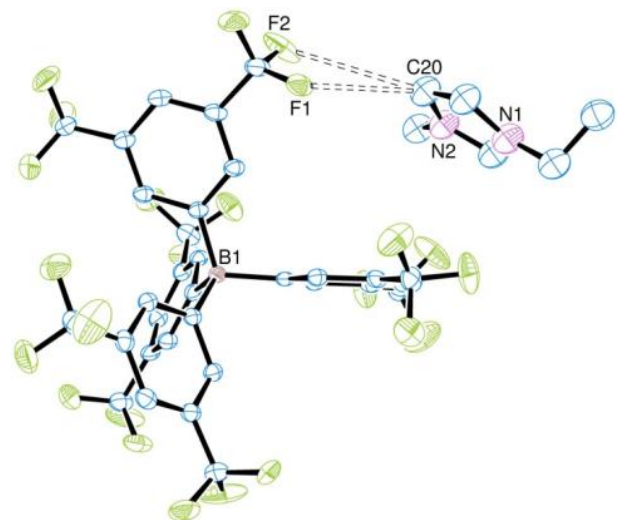

Figure 2: ORTEP representation of [EMIM][BAr $]$. Thermal ellipsoids are drawn at $50 \%$ probability and hydrogen atoms are omitted for clarity. The dashed lines represent the bifurcated hydrogen bonding through the $\mathrm{C} 4$ position (labelled $\mathrm{C20}$ ) of the imidazolium ring, including the medium-strength hydrogen bond to F1.

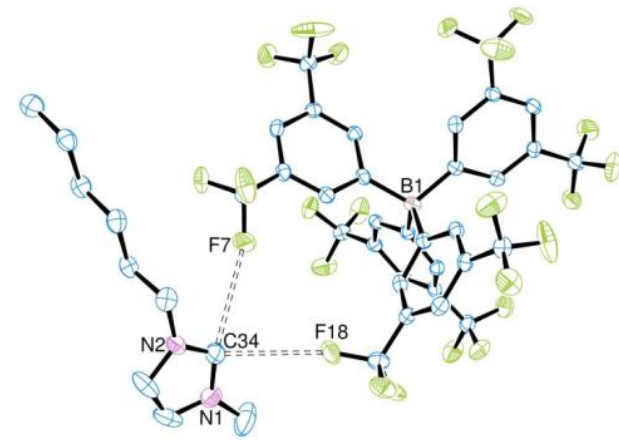

Figure 3: ORTEP representation of [HMIM][BAr $\left.{ }^{\mathrm{F}}\right]$. Thermal ellipsoids are drawn at 50\% probability and hydrogen atoms are omitted for clarity. The dashed lines represent the bifurcated hydrogen bonding through the $\mathrm{C} 2$ position (labelled $\mathrm{C} 34$ ) of the imidazolium ring.

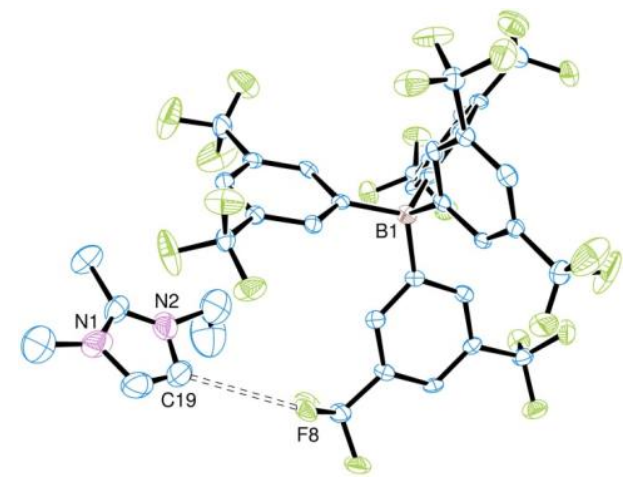


Figure 4: ORTEP representation of [EDMIM][BAr $\left.{ }^{\mathrm{F}}\right]$. Thermal ellipsoids are drawn at $50 \%$ probability and hydrogen atoms are omitted for clarity. The dashed line represents the shortest hydrogen bond through the $\mathrm{C} 4$ position (labelled C19) of the imidazolium ring.

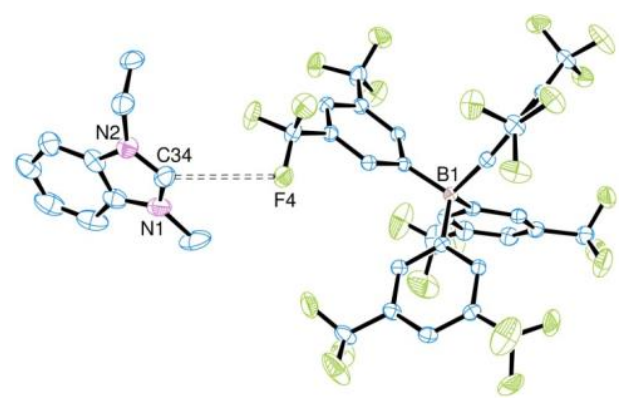

Figure 5: ORTEP representation of [EMBIM][BAr $\left.{ }^{\mathrm{F}}\right]$. Thermal ellipsoids are drawn at 50\% probability and hydrogen atoms are omitted for clarity. The dashed bond represents the hydrogen bond to the $\left[\mathrm{BAr}^{\mathrm{F}}\right]^{-}$ anion through the $\mathrm{C} 2$ position (labelled C34) of the benzimidazolium ring.

When examining the structures for hydrogen bonds, the criteria of Jeffrey and Steiner were used to classify the bonding into strong, moderate or weak hydrogen bonds. ${ }^{23}$ These criteria place a strong emphasis on the $\mathrm{D}-\mathrm{H} \cdots \mathrm{A}$ distances and angles: shorter $\mathrm{D} \cdots \mathrm{A}$ distances indicate stronger hydrogen bonds, as does the increasing linearity of the DHA angle. In all the compounds we studied no hydrogen bonds were consistent with the criteria for a "strong" hydrogen bond ( $\mathrm{D} \cdots \mathrm{A}<2.5 \AA \AA \mathrm{AHA}>170^{\circ}$ ), which is unsurprising given that strong hydrogen bonds typically involve molecules like water, protic acids or strong Lewis bases. For the ionic liquids containing WCAs, the hydrogen bonds are thus classified into "moderate" hydrogen bonds ( $D \cdots A$ between 2.5 and $3.2 \AA$; DHA > 130 ) or "weak" hydrogen bonds ( $D \cdots A>$ 3.2 $\AA$; DHA $<130^{\circ}$ ). This is consistent with a study by Deelman and co-workers, who found that increasing the amount of fluorination on a series of tetraphenylborate-based anions resulted in a significant reduction in the strength of hydrogen bonding between the $[\mathrm{BMIM}]^{+}$cation and the anions. ${ }^{24} \mathrm{~A}$ summary of the solid-state hydrogen bonding interactions for the structures containing the $\left.[\mathrm{BAr}]^{\mathrm{F}}\right]^{-}$anion is shown in Table 3.

\begin{tabular}{|l|l|l|}
\hline & \multicolumn{1}{|c|}{$\mathrm{H} 2$} & \multicolumn{1}{c|}{$\mathrm{H} 4 / 5$} \\
\hline EMIM & $3.45(1), 144.3$ & $3.046(9), 128.0$ \\
& $3.738(7), 122.0$ & $3.704(5), 149.0$ \\
& & $3.408(6), 162.7$ \\
\hline BMIM $^{\mathrm{a}}$ & $3.261(6), 138.5$ & $3.263(6), 123.6$ \\
& $3.41(1), 144.3$ & \\
\hline HMIM & $3.229(5), 128.0$ & $3.544(5), 140.0^{\mathrm{b}}$ \\
& $3.291(5), 131.4$ & $3.633(6), 145.8^{\mathrm{b}}$ \\
\hline EMBIM & $3.292(4), 136.7$ & $\mathrm{n} / \mathrm{a}$ \\
& $3.726(5), 148.3^{\mathrm{c}}$ & \\
\hline EDMIM & $\mathrm{n} / \mathrm{a}$ & $3.17(2), 152.0$ \\
& & $3.41(1), 163.0$ \\
& & $3.34(1), 118.3$ \\
\hline IMesH $^{\mathrm{d}}$ & $3.350(3), 138.2$ & $3.141(5), 100.3$ \\
\hline
\end{tabular}




\begin{tabular}{|l|l|l|}
\hline & & $3.28(1), 147.8$ \\
& & $3.26(1), 131.3$ \\
\hline IDiPPH & None & $3.343(2), 164.7$ \\
& & $3.297(3), 126.1$ \\
\hline
\end{tabular}

Table 3: hydrogen bonding summary for the $\left[\mathrm{BAr}{ }^{\mathrm{F}}\right]^{-}$-based ionic liquids. Distances $=\mathrm{C} \cdots \mathrm{X}(\mathrm{A})$, angles $=\mathrm{C}-$ $\mathrm{H}-\mathrm{X}\left({ }^{\circ}\right)$. Notes: $\mathrm{a}=$ data from literature; ${ }^{15 \mathrm{a}} \mathrm{b}=\mathrm{CH}-\pi$ interactions to a $\left[\mathrm{BAr}{ }^{\mathrm{F}}\right]^{-}$anion; $\mathrm{c}=$ to $\mathrm{Cl}_{\text {of }} \mathrm{CH}_{2} \mathrm{Cl}_{2}$ (lattice solvent); $d=$ data from literature. ${ }^{15 b}$

As the alkyl chain length increases from ethyl to $n$-hexyl, the $\mathrm{CH} \cdots \mathrm{X}$ distances through the most acidic proton $(\mathrm{H} 2)$ decrease markedly, implying an increase in the strength of the hydrogen bonding. This may be due to crystal packing effects where the longer alkyl chains disrupt the regular packing of the [BArF $]^{-}$ anions, allowing the cations to approach closer to the WCAs. It is notable that imidazolium-based salts with $\mathrm{n}$-alkyl chains $>\mathrm{C}_{12}$ have been observed to act as ionic liquid crystals with moderate $\mathrm{CH} \cdots \mathrm{F}$ hydrogen bonds $\left(\mathrm{CH} \cdots \mathrm{F}=2.950\right.$ (3) $\AA$ ), albeit to smaller WCAs such as $\left[\mathrm{PF}_{6}\right]^{-.25}$

All of the hydrogen bonds for the [EMIM], [BMIM] and [HMIM] salts reported here are classified as weak, except for the one through H4/5 of [EMIM][BArF]. Even though the bond angle is just below the cut-off for a moderate bond, at $3.05 \AA$ the $\mathrm{CH} \cdots \mathrm{F}$ distance is significantly within the limit to be classified as a moderate hydrogen bond. One other notable interaction is the backbone $\mathrm{H} 4 / 5$ hydrogen bonds from [HMIM] $\left[\mathrm{BAr}{ }^{\mathrm{F}}\right]$ which have an aryl ring from a $\left[\mathrm{BAr}^{\mathrm{F}}\right]^{-}$anion as the acceptor. These $\mathrm{CH} \cdots \pi$ interactions are very long and considerably longer than the average intermolecular $\mathrm{CH} \cdots \pi$ distance of 2.91(12) $\AA$ as calculated by Nishio et al., ${ }^{26}$ which is probably a result of the highly fluorinated nature of the aryl ring precluding closer contacts from occurring.

Altering the imidazolium ring to block some of the potential hydrogen bonding positions has a strong impact on the overall hydrogen bonding. Annulating a benzene ring on the backbone of the imidazolium ring removes the acidic $\mathrm{H} 4 / 5$ protons and the resulting 1-ethyl-3-methylbenzimidazolium cation only has $\mathrm{H} 2$ available to take part in hydrogen bonding. This results in two weak hydrogen bonds; one to the nearest $\left[\mathrm{BAr}{ }^{\mathrm{F}}\right]^{-}$anion whilst the other is to a lattice $\mathrm{CH}_{2} \mathrm{Cl}_{2}$ solvent, albeit this hydrogen bond is very weak. Conversely, methylating an imidazolium ring at the $\mathrm{C} 2$ position to form the [EDMIM] ${ }^{+}$cation prevents hydrogen bonding from occurring at the most acidic position. This results in the formation of one moderate hydrogen bond and two weak hydrogen bonds through the backbone $\mathrm{H} 4 / 5$ protons, an identical situation to that found for the $[\mathrm{EMIM}]^{+}$cation, indicating that blocking the $\mathrm{H} 2$ position does not materially affect the strength of the resulting hydrogen bonds through $\mathrm{H} 4$ and $\mathrm{H} 5$.

$\mathrm{N}$-heterocyclic carbenes (NHCs) with a large amount of steric bulk close to the carbene carbon have been widely used to isolate and stabilize very reactive species. The precursor to NHCs is generally an imidazolium salt and the steric bulk can also influence the ability of the imidazolium protons to take part in hydrogen bonding. [IMesH][BAr $]^{15 b}$ contains large mesityl groups bonded to the imidazolium ring. However, these are not sufficiently bulky to prevent all hydrogen bonding through the $\mathrm{H} 2$ proton, although only one weak hydrogen bond is present compared to two for the alkyl imidazolium cations such as [EMIM] $]^{+}$. Bulkier substituents, such as those found on [IDiPPH][BAr ${ }^{F}$, are capable of completely blocking all hydrogen bonds through $\mathrm{H} 2$. This was initially isolated as an unexpected hydrolysis product from the reaction of $\left[\mathrm{Ca}\left([18] \mathrm{aneO}_{4} \mathrm{~S}_{2}\right)(\mathrm{MeCN})_{2}\right]\left[\mathrm{BAr}^{\mathrm{F}}\right]_{2}{ }^{27}$ with the free NHC IDiPP ([18]aneO $\mathrm{O}_{4} \mathrm{~S}_{2}=1,4,10,13$ tetraoxa-7,16-dithiacyclooctadecane), but it can also be directly synthesized from [IDiPPH]Cl and 
$\mathrm{Na}\left[\mathrm{BAr}^{\mathrm{F}}\right]$. In each case, the steric bulk does not significantly affect the hydrogen bonding through the backbone $\mathrm{H} 4 / 5$ protons (Figure 6).

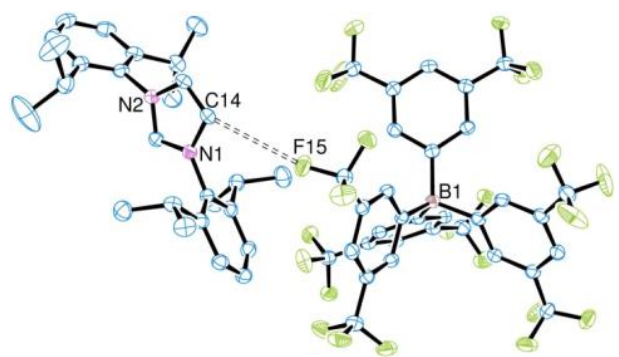

Figure 6: ORTEP representation of [IDiPPH][BAr $\left.{ }^{\mathrm{F}}\right]$. Thermal ellipsoids are drawn at 50\% probability and hydrogen atoms are omitted for clarity. The dashed line represents the shorter hydrogen bond through the backbone $\mathrm{C} 4$ position (labelled C14) of the imidazolium ring.

Using a cation with no acidic protons, such as [BMPYRR $]^{+}$(1-butyl-1-methylpyrrolidinium), results in an ionic liquid which contains no hydrogen bonding in either the solid or solution phase. Details of the solid state structure of [BMPYRR][BAr $\left.{ }^{\mathrm{F}}\right]$ are in the ESI.

Analogous compounds with the $\left[\mathrm{Al}\left(\mathrm{O}^{t} \mathrm{C}_{4} \mathrm{~F}_{9}\right)_{4}\right]^{-}$anion can be isolated in a similar manner, although X-ray quality crystals were only obtained for the $[E M I M]^{+}$and $[E D M I M]^{+}$salts (Figures 7 and 8 ). As for the $\left[\mathrm{BAr}^{\mathrm{F}}\right]^{-}$salts, ${ }^{1} \mathrm{H}$ NMR data indicated that there were no significant solution phase hydrogen bonds between cation and anion, but in the solid phase some weak hydrogen bonds were observed (Table 4).

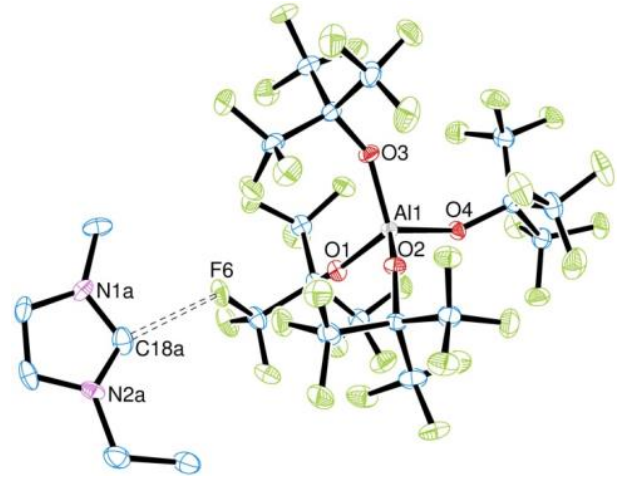

Figure 7: ORTEP representation of [EMIM] $\left[\mathrm{Al}\left(\mathrm{O}^{\mathrm{t}} \mathrm{C}_{4} \mathrm{~F}_{9}\right)_{4}\right]$. Thermal ellipsoids are drawn at $50 \%$ probability and hydrogen atoms are omitted for clarity. The dashed line represents the hydrogen bond through the C2 position (labelled C18a) of the imidazolium ring. 


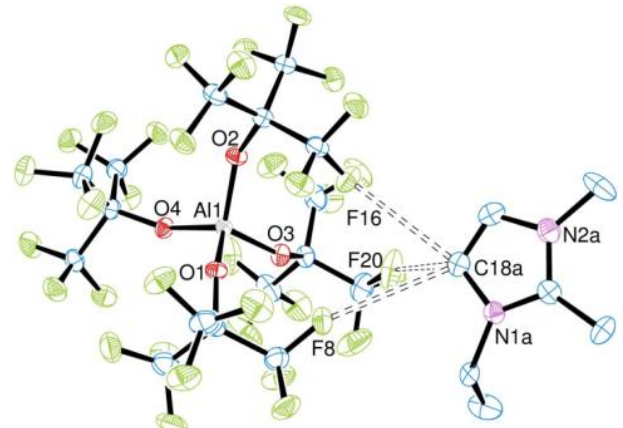

Figure 8: ORTEP representation of [EDMIM] $\left[\mathrm{AI}\left(\mathrm{O}^{\mathrm{t}} \mathrm{C}_{4} \mathrm{~F}_{9}\right)_{4}\right]$. Thermal ellipsoids are drawn at $50 \%$ probability and hydrogen atoms are omitted for clarity. The dashed lines represent the trifurcated hydrogen bonding interactions through the $\mathrm{C} 4$ position (labelled C18a) of the imidazolium ring.

\begin{tabular}{|l|l|l|}
\hline & \multicolumn{1}{|c|}{$\mathrm{H} 2$} & \multicolumn{1}{c|}{$\mathrm{H} 4 / 5$} \\
\hline EMIM & $3.34(6), 137.7$ & $3.368(7), 150.8$ \\
& & $3.269(7), 129.2$ \\
& & $3.20(4), 130.8$ \\
& & $3.37(4), 149.6$ \\
\hline EDMIM & n/a & $3.280(7), 138.2$ \\
& & $3.144(7), 127.9$ \\
& & $3.170(7), 124.6$ \\
\hline
\end{tabular}

Table 4: hydrogen bonding summary for the $\left[\mathrm{Al}\left(\mathrm{O}^{t} \mathrm{C}_{4} \mathrm{~F}_{9}\right)_{4}\right]^{-}$-based salts reported in this paper. Distances $=$ $\mathrm{C} \cdots \mathrm{F}(\AA)$, angles $=\mathrm{C}-\mathrm{H}-\mathrm{F}\left({ }^{\circ}\right)$.

Only one hydrogen bond was observed through the $\mathrm{H} 2$ proton of the $[\mathrm{EMIM}]\left[\mathrm{Al}\left(\mathrm{O}^{t} \mathrm{C}_{4} \mathrm{~F}_{9}\right)_{4}\right]$ salt. Although still classed as a weak hydrogen bond, the $\mathrm{CH} \cdots \mathrm{F}$ distance is notably shorter than either of the hydrogen bonds in the analogous [EMIM] [BArF] salt. There are four hydrogen bonds through the backbone H4/5 protons, with one meeting the criteria for a moderate strength hydrogen bond and two others showing strong directionality albeit they are slightly longer than $3.2 \AA$. For $[\mathrm{EDMIM}]\left[\mathrm{Al}\left(\mathrm{O}^{\mathrm{t}} \mathrm{C}_{4} \mathrm{~F}_{9}\right)_{4}\right]$ there are three hydrogen bonds through the backbone $\mathrm{H} 4 / 5$ protons (as in the [EMIM] analogue) with none meeting all of the criteria to be classed as moderate. However, one bond is significantly shorter than the distance cut-off at $3.144 \AA$ and only $2.1^{\circ}$ below the angle cut-off.

\section{Differential Scanning Calorimetry:}

SCFED processes are typically carried out at elevated temperatures, sometimes $>100{ }^{\circ} \mathrm{C}$, therefore all components of the electrolyte bath (including the background electrolyte) need to possess suitable thermal stability. ${ }^{11}$ Differential scanning calorimetry (DSC) was employed to examine the thermal behaviour of the ionic liquids. The $[\mathrm{EMIM}]^{+},[\mathrm{HMIM}]^{+},[\mathrm{EMBIM}]^{+}$and $[\mathrm{EDMIM}]^{+}$salts with both $\left[\mathrm{BAr}{ }^{\mathrm{F}}\right]^{-}$and $\left[\mathrm{Al}\left(\mathrm{O}^{\mathrm{t}} \mathrm{C}_{4} \mathrm{~F}_{9}\right)_{4}\right]^{-}$anions were heated to $200{ }^{\circ} \mathrm{C}$, cooled to $-50{ }^{\circ} \mathrm{C}$ and then reheated to $200{ }^{\circ} \mathrm{C}$ at a rate of $10{ }^{\circ} \mathrm{C} / \mathrm{min}$ to determine melting/freezing points (for [EMBIM] $\left[\mathrm{Al}\left(\mathrm{O}^{\mathrm{t}} \mathrm{C}_{4} \mathrm{~F}_{9}\right)_{4}\right]$ and [EDMIM] [Al $\left(\mathrm{O}^{\mathrm{t}} \mathrm{C}_{4} \mathrm{~F}_{9}\right)_{4}$ ] it was necessary to heat to $250{ }^{\circ} \mathrm{C}$ ). No evidence for decomposition or vaporization was observed within 
this temperature range. All samples used were crystalline solids and a typical DSC trace is shown in Figure 9:

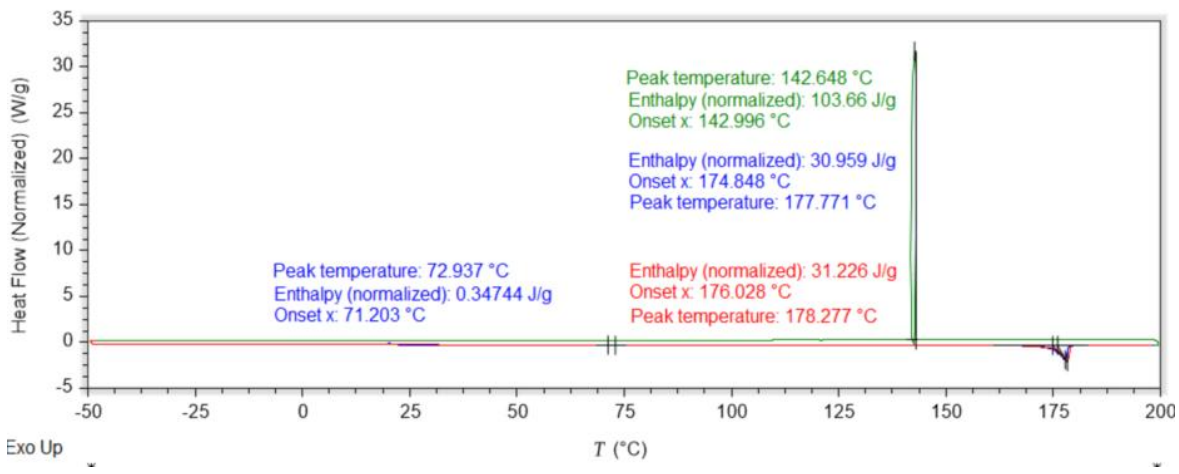

Figure 9: DSC trace for $[\mathrm{EMIM}]\left[\mathrm{Al}\left(\mathrm{O}^{\mathrm{t}} \mathrm{C}_{4} \mathrm{~F}_{9}\right)_{4}\right]$. Key: blue $=$ first heating cycle, green $=$ cooling cycle, red $=$ second heating cycle.

The data from the DSC traces are summarized in Table 5 and all eight DSC traces are shown in the ESI. Each compound showed a melting point in excess of $100{ }^{\circ} \mathrm{C}$ on the first heat cycle, apart from the two $[\mathrm{HMIM}]^{+}$salts which melted below $100{ }^{\circ} \mathrm{C}$ and can therefore be classified as room-temperature ionic liquids. ${ }^{28}$ This is consistent with the notion that longer alkyl chains on the imidazolium cation lead to lower melting points ( $c f \mathrm{C}_{12}$-based ionic liquids as liquid crystals). ${ }^{25}$ The observed melting points for [EMIM] [BAr $\left.{ }^{\mathrm{F}}\right]$ and $[\mathrm{HMIM}]\left[\mathrm{BAr} \mathrm{F}^{\mathrm{F}}\right]$ were in agreement with those reported by Nishi et al. ${ }^{10}$ Apart from [HMIM][BArF], which did not appear to solidify on the cooling cycle, the remaining seven samples all solidified with a substantial variation $\left(32-72^{\circ} \mathrm{C}\right)$ in the observed melting and freezing points. The exothermic process assigned as the freezing of [EMIM] [BArF] was only observed on the second heating cycle, and all seven compounds melted on the second cycle within $12{ }^{\circ} \mathrm{C}$ of the original melting temperature.

Several substantial endothermic processes were also observed and were assigned to phase changes. [EMBIM] $\left[\mathrm{BAr}^{\mathrm{F}}\right]$ exhibited a phase change at a similar temperature on both the first and second heating cycles indicating that on the cooling cycle the compound solidified into the same crystalline phase that was obtained from the chemical crystallisation process. None of the other compounds that exhibited a phase change showed this behaviour. Glass transitions on the cooling and heating cycles were also observed for [EMIM] $\left[\mathrm{BAr}^{\mathrm{F}}\right]$ and $[\mathrm{HMIM}]\left[\mathrm{BAr}^{\mathrm{F}}\right]$.

\begin{tabular}{|l|l|l|l|l|l|l|}
\hline & \multicolumn{2}{|c|}{ Heating 1 } & \multicolumn{2}{c|}{ Cooling } & \multicolumn{2}{c|}{ Heating 2 } \\
\hline & PC & \multicolumn{1}{c|}{ MP } & \multicolumn{1}{c|}{ GT } & \multicolumn{1}{c|}{ FP } & \multicolumn{1}{c|}{ GT } & \multicolumn{1}{c|}{ MP } \\
\hline$[\mathrm{EMIM}]\left[\mathrm{BAr}^{\mathrm{F}}\right]$ & 84.64 & 139.70 & -5.85 & $31.64^{\mathrm{a}}$ & -3.38 & 139.67 \\
\hline$\left.[\mathrm{EMIM}]\left[\mathrm{Al}^{(} \mathrm{OC}_{4} \mathrm{~F}_{9}\right)_{4}\right]$ & 72.93 & 174.85 & - & 142.68 & - & 178.28 \\
\hline$[\mathrm{HMIM}]\left[\mathrm{BAr}^{\mathrm{F}}\right]$ & - & 84.20 & -18.78 & n.o. & -17.50 & n.o. \\
\hline$[\mathrm{HMIM}]\left[\mathrm{Al}\left(\mathrm{OC}_{4} \mathrm{~F}_{9}\right)_{4}\right]$ & 47.47 & 67.94 & $-8.97^{\mathrm{b}}$ & 11.32 & & 68.03 \\
\hline
\end{tabular}




\begin{tabular}{|l|l|l|l|l|l|l|}
\hline$\left[\right.$ EMBIM] $\left[\mathrm{BAr}^{\mathrm{F}}\right]$ & 77.98 & 108.95 & - & 39.46 & $87.80^{\mathrm{b}}$ & 96.94 \\
\hline$[\mathrm{EMBIM}]\left[\mathrm{Al}\left(\mathrm{OC}_{4} \mathrm{~F}_{9}\right)_{4}\right]$ & - & 214.69 & - & 142.79 & - & 212.23 \\
\hline$[\mathrm{EDMIM}]\left[\mathrm{BAr}^{\mathrm{F}}\right]$ & 79.83 & 135.78 & - & 78.47 & - & 124.16 \\
\hline$[\mathrm{EDMIM}]\left[\mathrm{Al}\left(\mathrm{OC}_{4} \mathrm{~F}_{9}\right)_{4}\right]$ & - & 216.04 & - & 182.45 & - & 215.37 \\
\hline
\end{tabular}

Table 5: summary of the DSC data for the $\left[\mathrm{BAr}^{\mathrm{F}}\right]^{-}$and $\left[\mathrm{Al}\left(\mathrm{O}^{\mathrm{t}} \mathrm{C}_{4} \mathrm{~F}_{9}\right)_{4}\right]^{-}$-based ionic liquids with [EMIM] ${ }^{+}$, $[\mathrm{HMIM}]^{+},[\mathrm{EMBIM}]^{+}$and $[\mathrm{EDMIM}]^{+}$cations. All values in ${ }^{\circ} \mathrm{C} . \mathrm{PC}=$ phase change, $\mathrm{MP}=$ melting point, $\mathrm{GT}=$ glass transition, FP = freezing point. Notes: (a) observed on heating cycle 2; (b) phase change.

\section{Conclusions}

A systematic series of new ionic liquids containing the $\left[\mathrm{BAr}^{\mathrm{F}}\right]^{-}$and $\left[\mathrm{Al}\left(\mathrm{O}^{\mathrm{t}} \mathrm{C}_{4} \mathrm{~F}_{9}\right)_{4}\right]^{-}$weakly coordinating anions with imidazolium-based cations have been synthesized. In solution, ${ }^{1} \mathrm{H}$ NMR spectroscopy indicates that the ionic liquids are completely dissociated into discrete cations and anions, whilst in the solid phase weak hydrogen bonds exist between the acidic imidazolium protons and weakly coordinating anions. DSC data revealed that the ionic liquids are thermally stable to $200{ }^{\circ} \mathrm{C}$, therefore this class of compound could be suitable for use as a charge-carrying background electrolytes for SCFED processes at elevated temperatures. Additionally the salts based on $[\mathrm{HMIM}]^{+}$cations exhibited a melting point below $100{ }^{\circ} \mathrm{C}$, thus they meet the criteria to be classed as a room-temperature ionic liquid.

\section{Acknowledgements}

We would like to thank Drs Mateusz Pitak and Simon Coles for assistance with collecting the DSC data and Dr Mark Light for assistance with the X-ray structure solutions. This work was funded by EPSRC through a Programme Grant (EP/I033394/1) and through EP/K039466/1. The SCFED Project (www.scfed.net) is a multidisciplinary collaboration of British universities investigating the fundamental and applied aspects of supercritical fluids.

\section{Electronic Supplementary Information}

Spectroscopic data and complete DSC traces for all compounds examined in this study, and details of the solid state structure of $[\mathrm{BMPYRR}]\left[\mathrm{BAr}^{\mathrm{F}}\right]$, are included as electronic supplementary information. Crystallographic data in cif format have been deposited with the Cambridge Crystallographic Data Centre (CCDC) and given numbers CCDC 1514180-1514187. Copies of the data can be obtained free of charge from The Director, CCDC, 12 Union Road, Cambridge, CB2 1EZ, UK, fax: +44 1223 366033, e-mail: deposit@ccdc.cam.ac.uk or on the web at http://www.ccdc.cam.ac.uk.

\section{References}

1. (a) S. H. Strauss, Chem. Rev., 1993, 93, 927; (b) I. Krossing and I. Raabe, Angew. Chem. Int. Ed., 2004, 43, 2066.

2. (a) H. Nishida, N. Takada, M. Yoshimura, T. Sonoda, H. Kobayashi, Bull. Chem. Soc. Jpn., 1984, 57, 2600; (b) I. Krossing, Chem. Eur. J., 2001, 7, 490. 
3. S. D. Pike, A. L. Thompson, A. Algarra, D. C. Apperley, S. A. MacGregor and A. S. Weller, Science, 2012, 337, 1648.

4. T. A. Engesser, M. R. Lichtenthaler, M. Schleep and I. Krossing, Chem. Soc. Rev., 2016, 45, 789 and references therein.

5. (a) M. Everett, A. Jolleys, W. Levason, D. Pugh and G. Reid, Chem. Commun., 2014, 50, 5843; (b) M. Carravetta, M. Concistre, W. Levason, G. Reid and W. Zhang, Chem. Commun., 2015, 51, 9555; (c) M. J. D. Champion, J. M. Dyke, H. Bhakhoa, L. Rhyman, P. Ramasami, W. Levason, M. E. Light, D. Pugh and G. Reid, Inorg. Chem., 2015, 54, 2497.

6. M. J. D. Champion, W. Levason, D. Pugh and G. Reid, Dalton Trans., 2015, 44, 18748.

7. (a) P. Bartlett, D. Cook, M. George, A. Hector, W. Levason, G. Reid, D. Smith and W. Zhang, Phys. Chem. Chem. Phys., 2014, 16, 9202; (b) P. N. Bartlett, J. Burt, D. A. Cook, C. Y. Cummings, M. W. George, A. L. Hector, M. M. Hasan, J. Ke, W. Levason, D. Pugh, G. Reid, P. W. Richardson, D. C. Smith, J. Spencer and W. Zhang, Chem. Eur. J., 2016, 22, 302.

8. J. Ke, W. Su, S. M. Howdle, M. W. George, D. Cook, M. Perdjon-Abel, P. N. Bartlett, W. Zhang, F. Cheng, W. Levason, G. Reid, J. Hyde, J. Wilson, D. C. Smith, K. Mallik and P. Sazio, Proc. Natl. Acad. Sci. U. S. A., 2009, 106, 14768.

9. P. N. Bartlett, C. Y. Cummings, D. Pugh, G. Reid, W. Levason, M. M. Hasan, A. L. Hector, J. Spencer and D. C. Smith, J. Electrochem. Soc., 2015, 162, D619.

10. N. Nishi, S. Imakura and T. Kakiuchi, Anal. Chem., 2006, 78, 2726.

11. (a) P. N. Bartlett, D. C. Cook, M. W. George, J. Ke, W. Levason, G. Reid, W. T. Su and W. Zhang, Phys. Chem. Chem. Phys., 2010, 12, 492; (b) P. N. Bartlett, D. C. Cook, M. W. George, J. Ke, W. Levason, G. Reid, W. T. Su and W. Zhang, Phys. Chem. Chem. Phys., 2011, 13, 190; (c) H. Xue, N. Suleiman, J. Ke, W. Levason, D. Pugh, W. Zhang, G. Reid, P. Licence and M. W. George, Phys. Chem. Chem. Phys., 2016, 18, 14359.

12. P. N. Bartlett, C. Y. Cummings, W. Levason, D. Pugh and G. Reid, Chem. Eur. J., 2014, 20, 5019.

13. C. Y. Cummings, P. N. Bartlett, D. Pugh, G. Reid, W. Levason, M. M. Hasan, A. L. Hector, J. Spencer, D. C. Smith, S. Marks and R. Beanland, ChemElectroChem, 2016; 3, 726.

14. (a) A. Bösmann, G. Franciò, E. Janssen, M. Solinas, W. Leitner and P. Wasserscheid, Angew. Chem. Int. Ed., 2001, 40, 2697; (b) M. Solinas, A. Pfaltz, P. G. Cozzi and W. Leitner, J. Am. Chem. Soc., 2004, 126, 16142; (c) S. H. Jungbauer, S. M. Walter, S. Schindler, L. Rout, F. Kniep and S. M. Huber, Chem. Commun., 2014, 50, 6281; (d) V. Lecocq and H. Olivier-Bourbigou, Oil Gas Sci. Technol., 2007, 62, 761.

15. (a) J. Finden, G. Beck, A. Lantz, R. Walsh, M. J. Zaworotko and R. D. Singer, J. Chem. Cryst., 2003, 33, 287; (b) A. R. Kennedy, W. J. Kerr, R. Moir and M. Reid, Org. Biomol. Chem., 2014, 12, 7927 (c) S. M. Ivanova, B. G. Nolan, Y. Kobayashi, S. M. Miller, O. P. Anderson and S. H. Strauss, Chem. Eur. J., 2001, 7, 503; (d) T. Timofte, S. Pitula and A.-V. Mudring, Inorg. Chem., 2007, 46, 10938; (e) S. Bulut, P. Klose, M.-M. Huang, H. Weingärtner, P. J. Dyson, G. Laurenczy, C. Friedrich, J. Menz, K. Kümmerer and I. Krossing, Chem. Eur. J., 2010, 16, 13139.

16. B. Zhang, S. Li, M. Cokoja, E. Herdtweck, J. Mink, S.-L. Zang, W. A. Herrmann and F. E. Kühn, Z. Naturforsch, 2014, 69b, 1149.

17. (a) M. Brookhart, B. Grant and A. F. Volpe Jr., Organometallics, 1992, 11, 3920 ; (b) A. V. Astakhov, O. V. Khazipov, E. S. Degtyareva, V. N. Khrustalev, V. M. Chernyshev and V. P. Ananikov, Organometallics, 2015, 34, 5759; (c) L. Hintermann, Beilstein J. Org. Chem., 2007, 3, 22.

18. CrystalClear-SM Expert 3.1 b27, Rigaku Corporation, Tokyo, Japan, 2012; CrystalClear-SM Expert 2.1 b29, Rigaku Corporation, Tokyo, Japan, 2013.

19. L. J. Farrugia, J. Appl. Crystallogr., 2012, 45, 849. 
20. J. M. Dyke, W. Levason, M. E. Light, D. Pugh, G. Reid, H. Bhakhoa, P. Ramasami and L. Rhyman, Dalton Trans., 2015, 44, 13853.

21. F. H. Allen, O. Johnson, G. P. Shields, B. R. Smith and M. Towler, J. Appl. Crystallogr., 2004, 37, 335.

22. M. J. D. Champion, W. Levason, D. Pugh and G. Reid, New J. Chem., 2016, 40, 7181.

23. (a) G. A. Jeffrey, An Introduction to Hydrogen Bonding, Oxford University Press, Oxford, 1997; (b) T. Steiner, Angew. Chem., Int. Ed., 2002, 41, 48.

24. J. van den Broeke, M. Stam, M. Lutz, H. Kooijman, A. L. Spek, B.-J. Deelman and G. van Koten, Eur. J. Inorg. Chem., 2003, 2798.

25. C. M. Gordon, J. D. Holbrey, A. R. Kennedy and K. R. Seddon, J. Mater. Chem., 1998, 8, 2627.

26. M. Nishio, Y. Umezawa, K. Honda, S. Tsuboyama and H. Suezawa, CrystEngComm, 2009, 11, 1757.

27. W. Levason, D. Pugh, J. M. Purkis and G. Reid, Dalton Trans., 2016, 45, 7900.

28. K. N. Marsh, J. A. Boxall and R. Lichtenthaler, Fluid Phase Equilibr., 2004, 219, 93. 


\section{$\underline{\text { Table of Contents entry }}$}

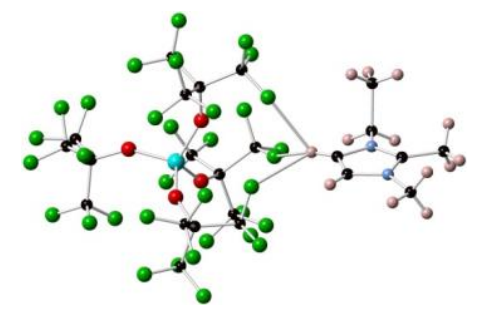

An investigation into the intramolecular interactions between a series of imidazolium salts and weakly coordinating anions reveals that it is possible to control the strength and location of hydrogen bonding by varying the substituents on the imidazolium cation. 